SAGA-HE-281

KEK-TH-1705

\title{
Evolution of vacuum fluctuations generated during and before inflation
}

\author{
Hajime Aoki $^{a}$, Satoshi Iso ${ }^{b c}$ and Yasuhiro Sekino ${ }^{b}$ \\ ${ }^{a}$ Department of Physics, Saga University, Saga 840-8502, Japan \\ ${ }^{b}$ KEK Theory Center, High Energy Accelerator Research Organization (KEK), \\ ${ }^{c}$ Graduate University for Advanced Studies (SOKENDAI), \\ Ibaraki 305-0801, Japan
}

\begin{abstract}
We calculate the time evolution of the expectation value of the energymomentum tensor for a minimally-coupled massless scalar field in cosmological spacetimes, with an application to dark energy in mind. We first study the evolution from inflation until the present, fixing the Bunch-Davies initial condition. The energy density of a quantum field evolves as $\rho \sim 3\left(H_{I} H\right)^{2} / 32 \pi^{2}$ in the matter-dominated (MD) period, where $H_{I}$ and $H$ are the Hubble parameters during inflation and at each moment. Its equation of state, $w=\rho / p$, changes from a negative value to $w=1 / 3$ in the radiation-dominated period, and from $1 / 3$ to $w=0$ in the MD period. We then consider possible effects of a Planckian universe, which may have existed before inflation, by assuming there was another inflation with the Hubble parameter $H_{P}\left(>H_{I}\right)$. In this case, modes with wavelengths longer than the current horizon radius are mainly amplified, and the energy density of a quantum field grows with time as $\rho \sim\left(a / a_{0}\right)\left(H_{P} H\right)^{2} / 32$ in the MD period, where $a$ and $a_{0}$ are the scale factors at each time and at present. Hence, if $H_{P}$ is of the order of the Planck scale $M_{P}, \rho$ becomes comparable to the critical density $3\left(M_{P} H\right)^{2}$ at the present time. The contribution to $\rho$ from the long wavelength fluctuations generated before the ordinary inflation has $w=-1 / 3$ in the free field approximation. We mention a possibility that interactions further amplify the energy density and change the equation of state.
\end{abstract}




\section{Introduction}

Our universe is well described by the so-called spatially-flat $\Lambda$ CDM model. Energy density of our universe is close to the critical density. According to the PLANCK 2013 results [1, only $5.1 \%$ of the energy density is attributed to a known form of baryonic matter, while $26.8 \%$ is attributed to cold dark matter (weakly interacting non-relativistic matter), and 68.3\% to dark energy (or the cosmological constant). Explaining the origin of these unknown ingredients, dark matter and dark energy, is one of the biggest challenges in modern physics.

There are various proposals for dark matter, such as supersymmetric particles, axions, and so on. Dark matter may well be one of these. However, the origin of dark energy is totally unclear. Although its equation of state, $w(=p / \rho)=-1$, seems like that of vacuum energy of quantum fields, there is no reasonable explanation for its magnitude, $\rho_{\mathrm{DE}}=3\left(M_{P} H_{0}\right)^{2} \Omega_{\Lambda} \sim(2.2 \mathrm{meV})^{4}$ with $\mathrm{meV}=10^{-3} \mathrm{eV}$. Here, $M_{P}=\left(8 \pi G_{N}\right)^{-1 / 2} \sim 2.4 \times 10^{30} \mathrm{meV}$ is the (reduced) Planck scale and $H_{0} \sim 1.4 \times 10^{-30} \mathrm{meV}$ is the current Hubble parameter. $\rho_{\mathrm{DE}}$ is far smaller than the expected magnitude of the vacuum energy $m^{4}$ in a theory with an ultra-violet (UV) cutoff, $m$, with any reasonable choice for $m$. If we take $m$ to be $M_{P}, \rho_{\mathrm{DE}}$ is smaller than $m^{4}$ by more than 120 orders of magnitude. Even if we take $m$ to be the supersymmetry breaking scale, electroweak scale, or any other natural scale in high-energy physics, $\rho_{\mathrm{DE}}$ is still much smaller than $m^{4}$. This is the cosmological constant problem [2]. It may turn out that the solution to this problem is given by the anthropic principle [3], but attempts at a dynamical explanation of dark energy are undoubtedly important.

In this paper, we study some aspects of vacuum energy of quantum fields in cosmological spacetimes, with an application to the cosmological constant problem in mind. Let us first note that the order of magnitude of the energy density of quantum fields is not necessarily given by the value of the UV cutoff. Expectation values of the energy-momentum tensor should be renormalized by subtracting the cutoff-dependent (divergent) term. There is a well-defined method for regularization and renormalization, which yields finite expectation values for energy-momentum tensors, which are covariant and conserved [4]. The terms to be subtracted are a combination of spacetime curvature tensors. Typical terms in the renormalized expectation value (such as the terms responsible for the Weyl anomaly) are of the order of the background curvature.

In particular, vacuum energy for fluctuations in the de Sitter background with the Hubble parameter $H$ is of the order $H^{4}$ (for massless fields), and has $w=-1$ [5, 6]. Since our present universe is close to de Sitter space, one may wonder if dark energy can be explained as vacuum energy in de Sitter with the 
current Hubble parameter $H_{0}$, but this does not seem to be plausible. Dark energy that we observe, $M_{P}^{2} H_{0}^{2}$, is much larger than the expected contribution from a single field, $H_{0}^{4}$.

However, local curvature is not the only dimensionful quantity that affects the renormalized energy-momentum tensor. To compute expectation values of fluctuations, we need to specify the vacuum state. This may depend on global properties of the geometry and the whole history of the universe; thus, different scales might be introduced in the problem.

There is by now strong evidence [7] that there has been a period of inflation in our past. The spacetime during inflation is nearly de Sitter space with the Hubble parameter $H_{I}$ being much larger than $H_{0}$. Inflation should have lasted long enough (e-foldings $N_{e} \gtrsim 60$ ) to solve the flatness and horizon problems. It would be reasonable to take the vacuum to be the Bunch-Davies vacuum [5] for de Sitter space with $H_{I}$. The Bunch-Davies vacuum is the one obtained by the Euclidean prescription, and reduces to the Minkowski vacuum in the short wavelength limit. Even if different initial states are taken, correlation functions will be attracted to those taken with respect to the Bunch-Davies vacuum, as shown quite generally [8].

Fluctuations of the massless scalar in the de Sitter background (in four spacetime dimensions) is of the order $H_{I}$, as is clear from dimensional analysis. Massless fluctuations are frozen (remain constant) outside the Hubble radius (see, e.g., [9]); thus, infra-red (IR) modes could have a large value in the universe after inflation. In fact, these fluctuations are considered to be the origin of the fluctuations of the cosmic microwave background (CMB) that is observed today [1, 7].

The purpose of this paper is to understand how the IR modes of quantum fields could affect the energy density in the present universe. We will mostly consider a massless minimally-coupled scalar field, and study the time evolution of the energy-momentum tensor in detail. Our result will shed light on the effect of almost massless and non-interacting fields, such as axions. Our work is related to the studies of the fluctuations of the graviton or the inflaton [10, 11, 12, but further modifications are necessary since energy-momentum tensors for gravitons have different tensor structures from those for scalars and those for the inflaton have contributions from the classical value of the field. We believe our work serves as a starting point for the study of the time evolution of those fields 1

\footnotetext{
${ }^{1}$ There is a paper [13], in which the authors claim that back reactions of gravitational waves produced during inflation can serve as a source for the acceleration of the present universe, by taking an approach somewhat different from ours.
} 
We will find that the magnitude of the present energy-momentum tensor of a massless field is of the order $H_{I}^{2} H_{0}^{2}$, with a possible factor logarithmic in the scale factor. The contribution from each momentum mode $k$ can be renormalized separately before integration over $k$ is performed. The equation of state for the (un-renormalized) contribution from each $k$ has $w>-1 / 3$ : The IR mode $k \rightarrow 0$ has $w \rightarrow-1 / 3$; for larger $k, w$ is larger, and the UV limit has $w \rightarrow 1 / 3$, which is the same as radiation. The equation of state may become $w<-1 / 3$ due to renormalization, but the terms arising from renormalization will be of the order of the background curvature. In the present universe, these will be of the order $H_{0}^{4}$, and are negligible 2

The value $H_{I}^{2} H_{0}^{2}$ is smaller than dark energy (or total energy density) in our universe, since the scale of inflation is smaller than the Planck scale, $H_{I} / M_{P}<$ $3.6 \times 10^{-5}$, as suggested by the observations of the CMB [7]. But, in this paper, we point out a possibility that the energy density of a quantum field takes a larger value 3 Even though the e-folding of inflation has to be large enough to make the region inside our horizon smooth, it does not have to be infinite. Finiteness of the e-foldings means the long wavelength modes have not been in causal contact during the inflationary era. There is no reason to expect that those modes are in the Bunch-Davies vacuum. It would not be too surprising if there are large fluctuations in the far IR. After all, the point of inflation is to push away inhomogeneities beyond our horizon. In particular, in the context of eternal inflation [17, 18, 19, our universe is generally surrounded by the region with a larger Hubble parameter where there are large fluctuations.

It is difficult to know what happened before inflation. In this paper, as an explicit example, we consider a double inflation model. We assume there was an inflation with the Hubble parameter $H_{P}$ of the order of the Planck scale $M_{P}$ (such as Starobinsky's inflation [20], for example), followed by either a radiationdominated or curvature-dominated transition period, before the usual inflation with $H_{I}$ starts. We fix the initial condition of the fields in the Planckian inflation period by taking the Bunch-Davies vacuum for de Sitter with the Hubble $H_{P}$, and study the time evolution afterwards. In this case, IR mode is enhanced to $H_{P}$.

\footnotetext{
${ }^{2}$ After submitting our paper, we became aware of a recent paper [14, which has substantial overlap with the first part of our paper. In [14], the authors study vacuum fluctuations of a massless minimally coupled test field in a background that evolves from inflation to radiation domination to matter domination. They use the approximation of sharp transition between these eras, just like we do. The results in the first part of our paper (which is summarized in this paragraph) is completely consistent with the ones in 14.

${ }^{3}$ This is different from proposals based on secular growth of fluctuations in inflation [15, 16]. We are not assuming the expectation value of energy density in pure de Sitter grows with time.
} 
By computing the evolution of the energy-momentum tensor for the double inflation model, we find the present value of vacuum energy to be of the order of $H_{P}^{2} H_{0}^{2}$. One may worry that the fluctuations become so large that they contradict the observed value of CMB fluctuations. This will depend on what field we are considering, and needs careful study. In this paper, we argue that it is possible to enhance the modes that have a longer wavelength than the scales that are observed in CMB, leaving shorter wavelength modes essentially unaffected.

The message of this paper is that vacuum energy of the order of the energy density of our present universe may arise, due to the enhancement of the IR fluctuations generated in the very early universe. In our analysis of free fields, we were able to obtain only $w>-1 / 3$, which cannot drive acceleration. However, we should mention that the free field approximation is not likely to be valid in the far IR where large fluctuations generated before the ordinary inflation exist. At the end of the paper, we will mention possible directions for future study to take interactions into account.

The paper is organized as follows. We review the basics of quantization of scalar fields in curved spacetimes in Section 2, After specifying the cosmic history of background geometry in Section 3, we obtain the wave function of a massless minimally-coupled scalar field, with the initial condition fixed in the inflationary era in Section 4. Then we calculate the energy-momentum tensor, paying special attention to the contributions from the IR modes in Section 5 . We explain the prescription for treating the UV divergence, and mention subtleties associated with a physical interpretation of cutoff depenent terms, and present the renormalized energy-momentum tensor in Section 6. We consider time evolution of the energy-momentum tensor from the inflationary era until the present in Section 17. In Section 8, we consider a double inflation model, and discuss the effects of a period that may have preceded the ordinary inflation. In section [8.2. we summarize time evolution of energy densities generated in the inflation period and in the pre-inflation period. They are shown in Figure 10. Section 9 is devoted to conclusions and discussion. In Appendix $\mathrm{A}$, we consider a double inflation model with a different intermediate stage. In Appendix B, we investigate IR behaviors of the wave functions.

\section{Scalar field in curved spacetimes}

In this section we briefly review some basics of the scalar field on curved spacetimes (see, for instance, ref. [4]).

A scalar field $\phi$ with a mass $m$ and a coupling $\xi$ to the scalar curvature $R$ 
in $n$-dimensional spacetime is described by the action

$$
\int d^{n} x(-g)^{1 / 2} \frac{1}{2}\left[g^{\mu \nu} \partial_{\mu} \phi \partial_{\nu} \phi-\left(m^{2}+\xi R\right) \phi^{2}\right],
$$

which gives the equation of motion

$$
\left[\square+m^{2}+\xi R\right] \phi=0
$$

The energy momentum tensor is given by

$$
\begin{aligned}
T_{\mu \nu}= & (1-2 \xi) \phi_{, \mu} \phi_{, \nu}+\left(2 \xi-\frac{1}{2}\right) g_{\mu \nu} g^{\rho \sigma} \phi_{, \rho} \phi_{, \sigma}-2 \xi \phi_{; \mu \nu} \phi \\
& +\frac{2}{n} \xi g_{\mu \nu} \phi \square \phi-\xi\left[R_{\mu \nu}-\frac{1}{2} R g_{\mu \nu}+\frac{2(n-1)}{n} \xi R g_{\mu \nu}\right] \phi^{2} \\
& +2\left[\frac{1}{4}-\left(1-\frac{1}{n}\right) \xi\right] m^{2} g_{\mu \nu} \phi^{2},
\end{aligned}
$$

where $\phi_{, \mu}=\partial_{\mu} \phi$ and $\phi_{; \mu \nu}=\nabla_{\nu} \partial_{\mu} \phi$. The conformally coupled scalar is described by $\xi=(n-2) /(4(n-1))$. In this paper we will study the minimally coupled scalar with $\xi=0$.

For background geometries, we consider Robertson-Walker spacetimes, which enjoy homogeneous and isotropic spaces, with the metric

$$
d s^{2}=a(\eta)^{2}\left[d \eta^{2}-\left(d x^{i}\right)^{2}\right] .
$$

$a(\eta)$ is the scale factor, and $\eta$ and $x^{i}$ are the conformal time and spacial coordinates. An explicit form of $a(\eta)$ will be specified in Section 3 ,

For quantizing the field, one expands the field as

$$
\phi\left(\eta, x^{i}\right)=\int \frac{d^{n-1} k}{(2 \pi)^{n-1}}\left[a_{\mathbf{k}} u_{\mathbf{k}}(\eta)+a_{-\mathbf{k}}^{\dagger} u_{-\mathbf{k}}(\eta)^{*}\right] e^{i \mathbf{k} \cdot \mathbf{x}}
$$

where the mode functions $u_{\mathbf{k}}(\eta)$ with the comoving momentum $\mathbf{k}$ are the solutions of the equation of motion (2.2), and are chosen to asymptote to positivefrequency modes in the remote past. A vacuum is then defined by $a_{\mathbf{k}}|0\rangle=0$. The vacuum $|0\rangle$, which is an in-state, evolves as $\eta$ increases, and if an adiabatic condition is broken the state gets excited above an adiabatic ground state at each moment, $\eta$.

In the Robertson-Walker spacetime (2.4), the wave equation (2.2) is written as

$$
\left[\partial_{\eta}^{2}+k^{2}+a^{2}\left(m^{2}+\left(\xi-\frac{n-2}{4(n-1)}\right) R\right)\right] \chi_{\mathbf{k}}=0
$$

where $k=\sqrt{\mathbf{k}^{\mathbf{2}}}$ and

$$
u_{\mathbf{k}}=a^{(2-n) / 2} \chi_{\mathbf{k}} .
$$


The expectation value of the energy momentum tensor in the state $|0\rangle$ is given by

$$
\left\langle 0\left|T_{\mu \nu}\left(\eta, x^{i}\right)\right| 0\right\rangle_{\mathrm{un}-\mathrm{ren}}=\int \frac{d^{n-1} k}{(2 \pi)^{n-1}} \mathcal{D}_{\mu \nu} u_{\mathbf{k}}(\eta) u_{\mathbf{k}}(\eta)^{*} .
$$

The right-hand side is obtained by inserting (2.5) into (2.3), and $\mathcal{D}_{\mu \nu}$ represents the differential operator that acts on $\phi^{2}$ in (2.3). The subscript 'un-ren' indicates that the UV divergence has not been subtracted yet. Regularization and renormalization will be discussed in Section 6. Note that (2.8) is independent of the space coordinates $x^{i}$, due to the spacial homogeneity of the RobertsonWalker spacetime.

\section{The background geometry}

Our universe is well approximated by the Robertson-Walker spacetime (2.4) in four spacetime dimensions, $n=4$. It experienced the inflation, radiationdominated (RD), and matter-dominated (MD) periods. We describe the three stages of the cosmic history by the following scale factor $a(\eta)$ :

$$
a(\eta)=\left\{\begin{array}{lll}
a_{\mathrm{Inf}}(\eta)=-\frac{1}{H_{I} \eta} & \left(-\infty<\eta<\eta_{1}<0\right) & \text { (Inflation) } \\
a_{\mathrm{RD}}(\eta)=\alpha \eta & \left(0<\eta_{2}<\eta<\eta_{3}\right) & (\mathrm{RD}) \\
a_{\mathrm{MD}}(\eta)=\beta \eta^{2} & \left(\eta_{4}<\eta<\eta_{0}\right) & (\mathrm{MD})
\end{array}\right.
$$

which is specified by the eight parameters $\left(H_{I}, \alpha, \beta, \eta_{1}, \eta_{2}, \eta_{3}, \eta_{4}, \eta_{0}\right)$. $H_{I}$ is the Hubble parameter in the inflation period. We show below that both $a$ and $a^{\prime}=\partial_{\eta} a$ must be continuous at the boundaries of the inflation-RD and the RD-MD periods. Then only four of the eight parameters are independent. As the four independent parameters, we will use $\left(H_{I}, a_{0}, H_{0}, z_{\text {eq }}\right)$ where $a_{0}$ and $H_{0}$ are the present scale factor 4 and the Hubble parameter, respectively, and $z_{\text {eq }}$ is the red-shift factor at the matter-radiation equality. All the eight parameters are written in terms of them.

The continuity condition of $a^{\prime}=\partial_{\eta} a$ can be easily understood as follows. The scale factor satisfies the Friedmann equation

$$
\begin{aligned}
& H^{2}=\frac{1}{3 M_{p}^{2}} \rho, \\
& \rho^{\prime}+3 a H(\rho+p)=0,
\end{aligned}
$$

where $\rho$ and $p$ are energy and pressure densities. The second equation demands that $\rho$ should be continuous unless the second term has a singularity. Then,

\footnotetext{
${ }^{4}$ We could set $a_{0}=1$, but we do not fix the value here.
} 
according to the first equation, $H=a^{\prime} / a^{2}$ is continuous and so is $a^{\prime}$ as well as $a$.

The continuity conditions between the inflation and RD periods are given by

$$
\begin{aligned}
& a_{\mathrm{Inf}}\left(\eta_{1}\right)=a_{\mathrm{RD}}\left(\eta_{2}\right):-\frac{1}{H_{I} \eta_{1}}=\alpha \eta_{2}, \\
& a_{\mathrm{Inf}}^{\prime}\left(\eta_{1}\right)=a_{\mathrm{RD}}^{\prime}\left(\eta_{2}\right) \quad: \quad \frac{1}{H_{I} \eta_{1}^{2}}=\alpha .
\end{aligned}
$$

They give the relation

$$
\eta_{1}=-\eta_{2}
$$

Similarly, the condition between the RD and MD periods is given by

$$
\begin{aligned}
& a_{\mathrm{RD}}\left(\eta_{3}\right)=a_{\mathrm{MD}}\left(\eta_{4}\right) \quad: \quad \alpha \eta_{3}=\beta \eta_{4}^{2}, \\
& a_{\mathrm{RD}}^{\prime}\left(\eta_{3}\right)=a_{\mathrm{MD}}^{\prime}\left(\eta_{4}\right) \quad: \quad \alpha=2 \beta \eta_{4},
\end{aligned}
$$

which lead to

$$
\eta_{3}=\frac{1}{2} \eta_{4}
$$

Now we determine $\left(a_{0}, H_{0}, z_{\mathrm{eq}}\right)$. The third equation of (3.1) gives

$$
\begin{aligned}
a_{0} & =\beta \eta_{0}^{2}, \\
H_{0} & =\frac{2}{\beta \eta_{0}^{3}},
\end{aligned}
$$

and we can write $\eta_{0}$ and $\beta$ in terms of $a_{0}$ and $H_{0}$ as

$$
\begin{aligned}
\eta_{0} & =2 H_{0}^{-1} a_{0}^{-1} \\
\beta & =\frac{1}{4} H_{0}^{2} a_{0}^{3} .
\end{aligned}
$$

Also, the same equation gives

$$
\frac{\eta_{0}}{\eta_{4}}=\sqrt{\frac{a_{0}}{a_{4}}}=\sqrt{1+z_{\mathrm{eq}}}
$$

and $\eta_{4}$ is written in terms of $z_{\text {eq }}$ and $\eta_{0}$ (hence, $a_{0}, H_{0}$ ). The other parameters can be similarly solved in terms of the four parameters. Here, we note a relation:

$$
\left(\frac{\eta_{3}}{\eta_{2}}\right)^{2}\left(\frac{\eta_{0}}{\eta_{4}}\right)^{3}=\frac{H_{I}}{H_{0}} .
$$

It can be proved straightforwardly from the above equations, and understood from the evolution of the Hubble parameters, $H \propto \eta^{-2}$ and $H \propto \eta^{-3}$ in the RD and MD periods, respectively, which are obtained by $H=a^{\prime} / a^{2}$ and (3.1). 
Finally, we estimate the numerical values of various parameters. $a_{0} \eta_{0}$ is determined by (3.12) with the use of the present Hubble:

$$
H_{0} \sim 67 \mathrm{~km} \mathrm{~s}^{-1} \mathrm{Mpc}^{-1} \sim 1.4 \times 10^{-30} \mathrm{meV} .
$$

Note that, as seen from (2.4), definition of $\eta$ has a rescaling ambiguity that can be absorbed into $a$, and only the combination of $\eta$ and $a$ has a physical meaning. $\eta_{4}$, and hence $\eta_{3}=\eta_{4} / 2$, is determined by (3.14) as

$$
\frac{\eta_{4}}{\eta_{0}} \sim \frac{1}{58} \sim 1.7 \times 10^{-2}
$$

where we used

$$
z_{\text {eq }} \sim 3.4 \times 10^{3}
$$

Similarly, $\eta_{2}$, and hence $\eta_{1}=-\eta_{2}$, is determined by (3.15) as

$$
\frac{\eta_{2}}{\eta_{0}}=\frac{\eta_{3}}{\eta_{4}} \sqrt{\frac{\eta_{0}}{\eta_{4}} \frac{H_{0}}{H_{I}}}>\frac{1}{2} \sqrt{58 \frac{1.4 \times 10^{-30} \mathrm{meV}}{8.8 \times 10^{25} \mathrm{meV}}} \sim 4.8 \times 10^{-28},
$$

where we have used the constraint from the CMB fluctuations:

$$
H_{I}<3.6 \times 10^{-5} M_{P} \sim 8.8 \times 10^{25} \mathrm{meV}
$$

As a final comment, we note that the higher derivatives of $a$ with respect to $\eta$ are not continuous. Consequently, we will see that the Bogoliubov coefficients have a long UV tail as a function of the momentum $k$. Such a long tail is an artifact of the rapid change of the scale factor and can be removed by smoothing the connections between the stages. It will be discussed in Section 6 .

\section{Time evolution of wave functions}

We now solve the wave equation (2.6) to obtain the wave function $\chi_{k}(\eta)$ in the cosmic history (3.1). In this paper we consider the minimally coupled case, $\xi=0$, in four dimensions, $n=4$. Then the wave equation (2.6) becomes

$$
\left[-\partial_{\eta}^{2}+\frac{1}{6} R a^{2}-m^{2} a^{2}\right] \chi_{\mathbf{k}}=k^{2} \chi_{\mathbf{k}}
$$

with

$$
\frac{1}{6} R a^{2}=\frac{a^{\prime \prime}}{a}=\left\{\begin{array}{lll}
2 / \eta^{2} & \left(\eta<-\left|\eta_{1}\right|\right) & \text { (Inflation) } \\
0 & \left(\left|\eta_{1}\right|<\eta<\eta_{4} / 2\right) & (\mathrm{RD}) \\
2 / \eta^{2} & \left(\eta_{4}<\eta<\eta_{0}\right) & (\mathrm{MD})
\end{array}\right.
$$

The relation with $u_{k}(2.7)$ becomes

$$
u_{\mathbf{k}}=\chi_{\mathbf{k}} / a
$$




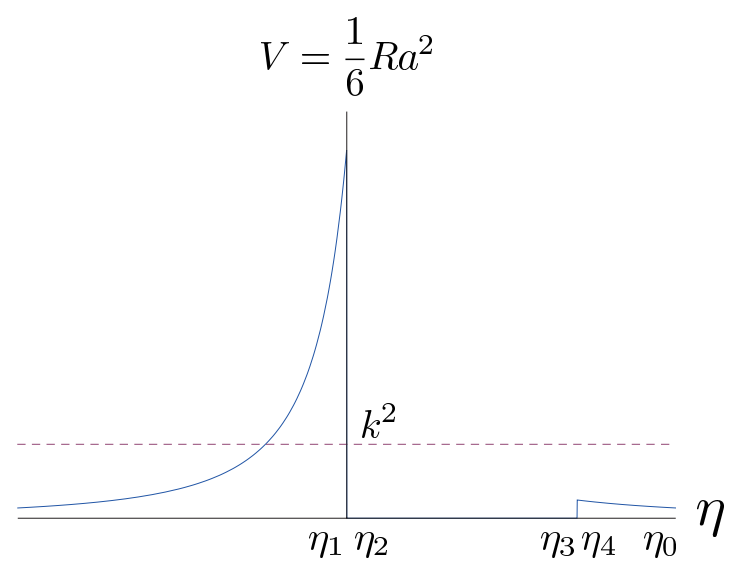

Figure 1: Potential for the wave equation (4.1) with $m=0$. The solid line depicts the potential (4.2), while the dashed line represents the right-hand side of (4.1). $\eta_{1}$ to $\eta_{4}$ are the boundaries of the inflation, RD, and MD periods. $\eta_{0}$ is the present time. Their numerical values are specified at the end of Section 3 . In particular, heights of the potential peaks are determined by (3.17) and (3.19). If we normalize the height of the potential at the present $\eta_{0}$ as 1 , the heights at $\eta=\eta_{1}$ and $\eta_{4}$ are given by $\lesssim 4.3 \times 10^{54}$ and $3.4 \times 10^{3}$, respectively.

Eq. (4.1) is interpreted as the time-independent Schrödinger equation for a onedimensional quantum system with a potential $V=R a^{2} / 6-m^{2} a^{2}$, by regarding $\eta$ as the spatial position. Figure 1 shows the potential (4.2) for the $m=0$ case. Note that it is discontinuous at the boundaries of the inflation, RD, and MD periods.

In the massless case, the solutions of (4.1) are given as

$$
\begin{aligned}
\chi_{\mathrm{Inf}, \mathbf{k}} & =\chi_{\mathrm{BD}, \mathbf{k}} \\
\chi_{\mathrm{RD}, \mathbf{k}} & =A(k) \chi_{\mathrm{PW}, \mathbf{k}}+B(k) \chi_{\mathrm{PW},-\mathbf{k}}^{*} \\
\chi_{\mathrm{MD}, \mathbf{k}} & =C(k) \chi_{\mathrm{BD}, \mathbf{k}}+D(k) \chi_{\mathrm{BD},-\mathbf{k}}^{*}
\end{aligned}
$$

in the inflation, $\mathrm{RD}$, and MD periods, respectively, where the wave functions are

$$
\begin{aligned}
\chi_{\mathrm{BD}, \mathbf{k}} & =\frac{1}{\sqrt{2 k}}\left(1-\frac{i}{k \eta}\right) e^{-i k \eta}, \\
\chi_{\mathrm{PW}, \mathbf{k}} & =\frac{1}{\sqrt{2 k}} e^{-i k \eta} .
\end{aligned}
$$

The wave function (4.4) contains only the positive frequency mode, which corresponds to taking the Bunch-Davies vacuum in the de Sitter spacetime. The constants $A, B, C$, and $D$ are easily determined by using the junction conditions 
of the wave functions, i.e., the continuity of $\chi$ and $\chi^{\prime}$. They become

$$
\begin{gathered}
\left(\begin{array}{c}
A(k) \\
B(k)
\end{array}\right)=\left(\begin{array}{c}
\left(1-\frac{i}{k \eta_{1}}-\frac{1}{2 k^{2} \eta_{1}^{2}}\right) e^{i k \eta_{2}} \\
\frac{1}{2 k^{2} \eta_{1}^{2}} e^{-i k \eta_{2}}
\end{array}\right) e^{-i k \eta_{1}} \\
\left(\begin{array}{c}
C(k) \\
D(k)
\end{array}\right)=\left(\begin{array}{cc}
\left(1+\frac{i}{k \eta_{4}}-\frac{1}{2 k^{2} \eta_{4}^{2}}\right) e^{i k\left(\eta_{4}-\eta_{3}\right)} & -\frac{1}{2 k^{2} \eta_{4}^{2}} e^{i k\left(\eta_{4}+\eta_{3}\right)} \\
-\frac{1}{2 k^{2} \eta_{4}^{2}} e^{-i k\left(\eta_{4}+\eta_{3}\right)} & \left(1-\frac{i}{k \eta_{4}}-\frac{1}{2 k^{2} \eta_{4}^{2}}\right) e^{-i k\left(\eta_{4}-\eta_{3}\right)}
\end{array}\right)\left(\begin{array}{l}
A(k) \\
B(k)
\end{array}\right) .
\end{gathered}
$$

These constants give the coefficients of the Bogoliubov transformations, and the Bunch-Davies vacuum is interpreted as an excited state on the adiabatic vacuum defined in the RD and MD periods, respectively 5 Let us look at the particle creation in the RD period. We expand $\phi\left(\eta, x^{i}\right)$ in terms of the adiabatic wave functions $\chi_{\mathrm{PW}}$ at each moment in the RD period as

$$
\phi\left(\eta, x^{i}\right)=\frac{1}{a} \int \frac{d^{3} k}{(2 \pi)^{3}}\left[a_{\mathbf{k}}^{\mathrm{ad}} \chi_{\mathrm{PW}}(\eta, k)+a_{-\mathbf{k}}^{\mathrm{ad} \dagger} \chi_{\mathrm{PW}}(\eta, k)^{*}\right] e^{i \mathbf{k} \cdot \mathbf{x}} .
$$

The adiabatic vacuum is defined by $a_{\mathbf{k}}^{\text {ad }}\left|0_{\mathrm{ad}}\right\rangle=0$. If we start from the BunchDavies vacuum defined by $a_{\mathbf{k}}|0\rangle=0$, the state $|0\rangle$ evolves into a highly excited state on the adiabatic vacuum $\left|0_{\mathrm{ad}}\right\rangle$. By using the solutions of the wave equation and comparing (2.5) and (4.11), we have

$$
\left(\begin{array}{c}
a_{\mathbf{k}}^{\mathrm{ad}} \\
a_{-\mathbf{k} \dagger}^{\mathrm{ad}}
\end{array}\right)=\left(\begin{array}{ll}
A(k) & B^{*}(k) \\
B(k) & A^{*}(k)
\end{array}\right)\left(\begin{array}{c}
a_{\mathbf{k}} \\
a_{-\mathbf{k}}^{\dagger}
\end{array}\right) .
$$

The coefficients $A(k), B(k)$ satisfy the relation $|A(k)|^{2}-|B(k)|^{2}=1$. Furthermore, we can always make $A(k)$ real by redefining the phase of $a_{\mathbf{k}}$. So the coefficients can be parametrized as

$$
A(k)=\cosh \theta_{k}, \quad B(k)=-\sinh \theta_{k} e^{i \phi_{k}} .
$$

The inverse of the transformation (4.12) is generated by using the squeezing operator

$$
G_{k}=a_{\mathbf{k}}^{\mathrm{ad}} a_{-\mathbf{k}}^{\mathrm{ad}} e^{i \phi_{k}}-a_{\mathbf{k}}^{\mathrm{ad} \dagger} a_{-\mathbf{k}}^{\mathrm{ad} \dagger} e^{-i \phi_{k}}
$$

as

$$
\left(\begin{array}{c}
a_{\mathbf{k}} \\
a_{-\mathbf{k}}^{\dagger}
\end{array}\right)=e^{\theta_{k} G_{k}}\left(\begin{array}{c}
a_{\mathbf{k}}^{\mathrm{ad}} \\
a_{-\mathbf{k}}^{\mathrm{ad}}
\end{array}\right) e^{-\theta_{k} G_{k}} .
$$

\footnotetext{
${ }^{5}$ In a recent paper 21, particle content and the degree of classicality were studied in a background similar but slightly different from ours (de Sitter in inflation, followed by radiation dominance, and a late time de Sitter).
} 
The Bunch-Davies vacuum is then written as

$$
|0\rangle=\prod_{k} e^{\theta_{k} G_{k}}\left|0_{\mathrm{ad}}\right\rangle
$$

Since the squeezing operator $G_{k}$ is bilinear in the creation operators, the BunchDavies vacuum is a collection of excited states with multiple pairs of particles. Concretely, $|0\rangle$ can be expanded as

$$
\begin{aligned}
|0\rangle & =\prod_{k} \frac{1}{\cosh \theta_{k}} e^{-\tanh \theta_{k} e^{-i \phi_{k}} a_{\mathbf{k}}^{\mathrm{ad} \dagger} a_{-\mathbf{k}}^{\mathrm{ad} \dagger}\left|0_{\mathrm{ad}}\right\rangle} \\
& =\prod_{k} \frac{1}{\cosh \theta_{k}} \sum_{n=0}^{\infty}\left(-\tanh \theta_{k} e^{i \phi_{k}}\right)^{n}|n\rangle_{k} \otimes|n\rangle_{-k} .
\end{aligned}
$$

The number of created particles is calculated as

$$
\left\langle 0\left|a_{\mathbf{k}}^{\mathrm{ad} \dagger} a_{\mathbf{k}}^{\mathrm{ad}}\right| 0\right\rangle=\left(\sinh \theta_{k}\right)^{2}=|B(k)|^{2}=\frac{1}{4 k^{4} \eta_{1}^{4}} .
$$

Hence, it becomes very large for $k \ll 1 / \eta_{1}$. The same calculation is performed in the MD period, and the number of created particles is given by $|D(k)|^{2}$.

Now, let us investigate the IR, i.e., small- $k$, behavior of the wave functions. When $k|\eta| \ll 1$, (4.7) behaves as $\chi_{\mathrm{Inf}}=\chi_{\mathrm{BD}} \sim k^{-3 / 2}$. Terms of $\mathcal{O}\left(k^{-1 / 2}\right)$ cancel and the next term starts with $k^{1 / 2}$. By using (4.3) with (3.1), the IR behavior of $u$ becomes

$$
u_{\mathrm{Inf}}^{\mathrm{IR}}=\frac{i}{\sqrt{2}} H_{I} k^{-3 / 2}+\mathcal{O}\left(k^{1 / 2}\right)
$$

in the inflation period. This IR behavior of the wave function is kept until the $\mathrm{RD}$ and MD periods. It generally holds that the leading part of the superhorizon modes of a massless field is time-independent, i.e., frozen. It gives the seeds of the CMB fluctuations. (See, for instance, sections 7.3.2, 8.4, and 9.9 of ref. [9.)

Let us confirm the above IR behavior of the wave function. Since $A(k)$ and $B(k)$ in (4.9) are proportional to $k^{-2}$ in the IR region for $k \ll\left|\eta_{1}\right|^{-1}$, the wave function in the $\mathrm{RD}$ period (4.5) seems to behave $\chi_{\mathrm{RD}}(k) \sim k^{-5 / 2}$ for $k \ll \eta^{-1},\left|\eta_{1}\right|^{-1}$. Similarly, the behavior $C, D \sim k^{-4}$ indicates much more violent IR behavior, $\chi_{\mathrm{MD}} \sim k^{-11 / 2}$, for $k \ll \eta^{-1},\left|\eta_{1}\right|^{-1}, \eta_{4}^{-1}$. However, lots of cancellations occur and the IR behavior becomes milder. Indeed, in the RD period, (4.9) is rewritten as

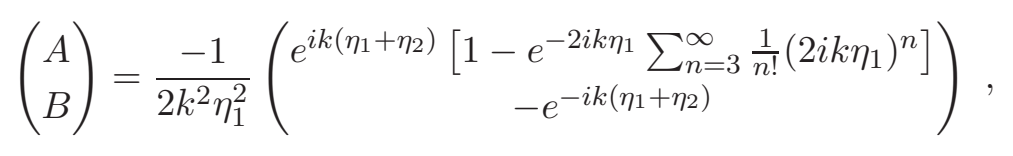


where the terms with $k$ and the terms with $k^{2}$ cancel in the square bracket. Then the wave function (4.5) behaves as

$$
\begin{aligned}
\chi_{\mathrm{RD}}^{\mathrm{IR}} & =\frac{1}{\sqrt{2 k}}\left(\frac{2 i}{2 k^{2} \eta_{1}^{2}} \sin \left(k\left(\eta-\eta_{1}-\eta_{2}\right)\right)+\mathcal{O}\left(k \eta_{1}\right) \cdot e^{-i k\left(\eta-\eta_{1}-\eta_{2}\right)}\right) \\
& =\frac{i}{\sqrt{2}} \frac{\eta-\eta_{1}-\eta_{2}}{\eta_{1}^{2}} k^{-3 / 2}+\mathcal{O}\left(k^{1 / 2}\right)
\end{aligned}
$$

in the IR regions. Note that the terms with $k^{-1 / 2}$ cancel each other and the next terms start with $k^{1 / 2}$. Moreover, by using $\eta_{1}=-\eta_{2}$ in (3.6), the leading term in (4.22) gives

$$
u_{\mathrm{RD}}^{\mathrm{IR}}=\frac{\chi_{\mathrm{RD}}^{\mathrm{IR}}}{a_{\mathrm{RD}}}=\frac{i}{\sqrt{2}} \frac{\eta}{\eta_{1}^{2}} k^{-3 / 2} \frac{H_{I} \eta_{1}^{2}}{\eta}=\frac{i}{\sqrt{2}} H_{I} k^{-3 / 2},
$$

where we used (3.1) and (3.5). Hence, the IR behavior (4.20) is shown to be maintained in the RD period as well.

As we show in Appendix B.1, the same IR behavior holds in the MD period.

\section{$5 \quad$ Energy-momentum tensors}

For a minimally-coupled massless scalar in four dimensions, i.e., $\xi=0, m=0$, and $n=4$, the energy density $\rho=\left\langle T_{\eta}^{\eta}\right\rangle$ and the pressure density $p=-\left\langle T_{i}^{i}\right\rangle(i$ is not summed over) are given from (2.3) by

$$
\begin{aligned}
\rho(\eta)^{\mathrm{un}-\mathrm{ren}} & =\frac{1}{a^{2}} \int \frac{d^{3} k}{(2 \pi)^{3}} \frac{1}{2}\left[\left|u_{k}^{\prime}(\eta)\right|^{2}+k^{2}\left|u_{k}(\eta)\right|^{2}\right] \\
& =\frac{1}{4 \pi^{2} a^{4}} \int d k k^{2}\left[\left|\chi^{\prime}\right|^{2}-\frac{a^{\prime}}{a}\left(\chi^{*} \chi^{\prime}+\chi^{\prime *} \chi\right)+\left(\left(\frac{a^{\prime}}{a}\right)^{2}+k^{2}\right)|\chi|^{2}\right] \\
p(\eta)^{\mathrm{un}-\mathrm{ren}} & =\frac{1}{a^{2}} \int \frac{d^{3} k}{(2 \pi)^{3}} \frac{1}{2}\left[\left|u_{k}^{\prime}(\eta)\right|^{2}-\frac{1}{3} k^{2}\left|u_{k}(\eta)\right|^{2}\right] \\
& =\frac{1}{4 \pi^{2} a^{4}} \int d k k^{2}\left[\left|\chi^{\prime}\right|^{2}-\frac{a^{\prime}}{a}\left(\chi^{*} \chi^{\prime}+\chi^{\prime *} \chi\right)+\left(\left(\frac{a^{\prime}}{a}\right)^{2}-\frac{1}{3} k^{2}\right)|\chi|^{2}\right] .
\end{aligned}
$$

Here, the expectation values are taken in the Bunch-Davies vacuum. The superscript 'un-ren' means that the UV divergences are not yet subtracted.

We first examine the contributions from the IR modes. By substituting the IR behavior of the wave function (4.20), which is kept until the RD and MD 
periods, into (5.1) and (5.3), one obtains

$$
\begin{aligned}
\rho^{\mathrm{IR}} & =\frac{H_{I}^{2}}{8 \pi^{2} a^{2}} \int_{0} d k\left[k+\mathcal{O}\left(k^{3}\right)\right], \\
p^{\mathrm{IR}} & =\frac{H_{I}^{2}}{8 \pi^{2} a^{2}} \int_{0} d k\left[-\frac{1}{3} k+\mathcal{O}\left(k^{3}\right)\right] .
\end{aligned}
$$

Since the IR wave function (4.20) is frozen and time-independent, only the spacial derivative terms $k^{2}\left|u_{k}(\eta)\right|^{2}$ contribute to $\rho^{\mathrm{IR}}$ and $p^{\mathrm{IR}} 6$ Due to the amplification of the wave function (4.20), $\rho^{\mathrm{IR}}$ and $p^{\mathrm{IR}}$ are enhanced to the order of $H_{I}^{2}$. The leading term in (5.5) and (5.6) gives $w^{\mathrm{IR}}=p^{\mathrm{IR}} / \rho^{\mathrm{IR}}=-1 / 3$.

The two-point correlation function of massless fields receives a logarithmic IR growth:

$$
\begin{aligned}
\lim _{x \rightarrow y}\langle 0|\phi(\eta, x) \phi(\eta, y)| 0\rangle & =\lim _{x \rightarrow y} \int \frac{d^{3} k}{(2 \pi)^{3}} u_{k}(\eta) u_{k}(\eta)^{*} e^{i k \cdot(x-y)} \\
& \sim \frac{H_{I}^{2}}{4 \pi^{2}} \int d k \frac{1}{k} .
\end{aligned}
$$

In contrast, the energy and pressure densities, (5.5) and (5.6), are IR finite since they involve derivatives, and the IR divergences are canceled. This is due to the fact that for an exactly massless field, the constant part of the field (which gives rise to the logarithmic divergence) does not have a physical meaning.

However, if we take the massless limit of a massive field, the mass term in the energy-momentum tensor gives a non-vanishing contribution, since $\left\langle\phi^{2}\right\rangle \sim$ $H_{I}^{4} / m^{2}$. This can be computed by using the exact wave function for massive fields, which is written in terms of Hankel functions in de Sitter space [4, 5, or can be seen as follows. We compute

$$
\rho(\eta)_{\text {mass }}=\int \frac{d^{3} k}{(2 \pi)^{3}} \frac{m^{2}}{2}\left|u_{k}(\eta)\right|^{2} .
$$

By introducing a small mass, the potential in the inflation period changes from $2 / \eta^{2}$ to $\left(2-\left(m / H_{I}\right)^{2}\right) / \eta^{2}$, and the IR behavior of the massless wave function (4.20) is modified to

$$
u_{\mathrm{Inf}}^{\mathrm{IR}}(\eta) \sim \frac{i}{\sqrt{2}} H_{I} k^{-3 / 2}(k|\eta|)^{\frac{\left(m / H_{I}\right)^{2}}{3}} .
$$

Inserting it into (5.8), we obtain

$$
\rho(\eta)_{\text {mass }}=\frac{m^{2}}{2} \int_{0}^{1 /|\eta|} \frac{d k}{2 \pi^{2}} \frac{H_{I}^{2}}{2 k}(k|\eta|)^{\frac{2\left(m / H_{I}\right)^{2}}{3}}=\frac{3 H_{I}^{4}}{16 \pi^{2}} .
$$

\footnotetext{
${ }^{6}$ The absence of $\mathcal{O}\left(k^{-1 / 2}\right)$ term in the IR behavior of wave function (4.20) is important to assure the robustness of (5.5) and (5.6). If $\mathcal{O}\left(k^{-1 / 2}\right)$ term existed, it would change the coefficient of $\mathcal{O}(k)$ term in (5.5) through the $\left|u_{k}^{\prime}(\eta)\right|^{2}$ term in (5.1).
} 
Here, the integration is performed up to $k=1 /|\eta|$, which corresponds to the horizon scale $k_{\text {phys }}=k / a_{\text {Inf }}=H_{I}$, since the IR behavior (5.9) is valid below this scale. The result (5.10) is independent of the mass. Hence, the massless limit of a massive theory gives an additional contribution to the expectation value of the energy-momentum tensor. But we should note that, in order to obtain the contribution, we implicitly assumed that there is no other IR cutoff. If there exists a physical IR cutoff, $\Lambda_{\mathrm{IR}}$, the logarithmic IR divergence is automatically cured by $\Lambda_{\mathrm{IR}}$ and no such singular behavior with $1 / \mathrm{m}^{2}$ appears. Such an IR cutoff may be given, e.g., by the initial time of the inflation period. In that case, the expectation value of a massless scalar $\left\langle\phi(\eta, x)^{2}\right\rangle$ will be proportional to the physical time interval since the initial time [22, 23, 24, 25], and will not be infinite 7 Then $\rho_{\text {mass }}$ vanishes in the $m \rightarrow 0$ limit and the massless limit of a massive theory gives the same answer as the purely massless theory. In the following analysis of the present paper, we assume an existence of such an IR physical cutoff. A further possible contribution of $\rho_{\text {mass }}$ to the vacuum energy will be investigated in a separate paper.

\section{Renormalized energy-momentum tensors}

The energy and pressure densities (5.5) and (5.6) are UV divergent, and must be regularized and renormalized. This has been studied extensively in the past (see, for instance, section 6 of ref. [4]), so we will keep the description brief and just present the result.

We first make the integral finite by using one of the regularization prescriptions, such as the dimensional regularization or the covariant point-splitting. We then perform renormalization by subtracting the terms that can be absorbed by redefinitions of coupling constants in the gravity action that have the dimensions four (the cosmological constant), two (the Newton constant inverse), and zero (the coefficients for curvature tensor squared terms). In general, the renormalized energy-momentum tensor takes the form

$$
\left\langle T_{\mu \nu}\right\rangle_{\mathrm{ren}}=\mathcal{D}_{\mu \nu}\left[G-G^{(A)}\right]
$$

where $\mathcal{D}_{\mu \nu}$ stands for the derivative operators in (2.8). $G$ is the two-point correlation function obtained in the previous sections. $G^{(A)}$ is the subtraction term, which is the two-point function obtained using the first few orders of the DeWitt-Schwinger expansion [30]. This is an expansion around the flat space-

\footnotetext{
${ }^{7}$ See $[26,27,28,29$, for recent studies on this linear growth of perturbation in inflation and the effect of the initial time cutoff.
} 
time, and the expansion depends only on the information of local geometry, namely, the subtraction terms are written in terms of curvature tensors.

Another way of obtaining subtraction terms, which has been shown to be equivalent to the DeWitt-Schwinger expansion in various cases, including the Friedmann-Robertson-Walker (FRW) universe, is the adiabatic regularization [31, 32. In this scheme, $G^{(A)}$ is obtained by using the WKB expansion of the mode functions up to the adiabatic order four. With this prescription, the subtraction can be performed at each $k$ separately, and we can obtain finite integrals without the need of an explicit regularization. In this sense, the adiabatic regularization is a prescription for subtraction, not a regularization method.

The subtraction term contains an IR divergent term for massless theories; thus, the renormalized energy-momentum tensor should be defined by taking the massless limit of a massive theory. The pieces that remain finite as a result of this procedure are given, e.g., in Eqs. (3.14) and (3.15) of [32] for the general FRW universe. The Weyl anomaly arises from this procedure. However, since these contributions are smaller than the terms that we are mainly interested in, we will ignore these contributions in the analyses of the paper.

Before starting the analysis of the renormalized energy-momentum tensor in our background, we would like to make a side remark about the UV divergent (cutoff-dependent) terms. We emphasize here that a physical interpretation of these terms is a very subtle issue. For example, consider the quartically divergent term in the effective action, which is the leading divergence in four spacetime dimensions,

$$
\mathcal{L}_{\text {eff }, 4}=-\sqrt{-g} k_{\mathrm{UV}}^{4}
$$

where $k_{\mathrm{UV}}$ is the UV cutoff for the momentum. This term is often interpreted as a contribution from quantum fields to the cosmological constant.

On the other hand, if one computes $\left\langle T_{\mu \nu}\right\rangle$, the quartically divergent term has $w=1 / 3$, which is the equation of state for radiations, not the cosmological constant. This is indeed expected from the fact that in the limit of high momentum, there is no particle creation, and the field behaves as a collection of radiations. One can also see $w=1 / 3$ directly from the coefficients of the $k^{3}$ terms in the integrand of (6.4) and (6.5) below.

It is intuitively unclear how these two different equations of state for the quartically divergent terms are consistent with each other. The argument in the literature [32] is based on the dimensional regularization: The quartic divergence in $\left\langle T_{\mu \nu}\right\rangle$ is regularized, then one looks at the contribution at the pole $1 /(n-4)$ and finds that it is proportional to $g_{\mu \nu}$. Thus, it is removed by renormalizing the cosmological constant. 
The above difference of equation of state in the two approaches may be attributed to the fact that the UV cutoff $k_{\mathrm{UV}}$ itself depends on the metric. One can introduce a UV cutoff by putting the fields at two points separated by a coordinate time interval $\Delta t$ (though we expect that the details of the regularization will not affect the conclusion). Then $k_{\mathrm{UV}}$ will depend on $g_{00}$; we assume it is of the noncovariant form $k_{\mathrm{UV}}^{2}=\left(g_{00}(\Delta t)^{2}\right)^{-1}$. By taking this into account 8 the energy-momentum tensor for (6.2) becomes

$$
\begin{aligned}
\left\langle T_{\mu \nu}\right\rangle_{4} & =\frac{2}{\sqrt{-g}} \frac{\delta \mathcal{L}_{\mathrm{eff}, 4}}{\delta g^{\mu \nu}} \\
& =k_{\mathrm{UV}}^{4} g_{\mu \nu}-4 k_{\mathrm{UV}}^{4} \frac{g_{0 \mu} g_{0 \nu}}{g_{00}},
\end{aligned}
$$

where the second term comes from the variation of $k_{\mathrm{UV}}^{4}$ with respect to $g^{00}$. The expression (6.3) indeed has $w=1 / 3$ when the background metric is diagonal.

In our opinion, it could be misleading to discuss the cosmological constant problem by looking at cutoff-dependent quantities with UV power divergences 9 since the subtracted term is not generally covariant. In this paper, we will always consider the renormalized quantities when we discuss physical effects.

\subsection{RD period}

Let us now evaluate $\rho$ and $p$ for the state (4.5) in the RD period. By substituting the wave function (4.5) into (5.2) and (5.4), one obtains

$$
\begin{aligned}
\rho_{\mathrm{RD}}^{\mathrm{un}-\mathrm{ren}}= & \frac{1}{8 \pi^{2} a^{4}} \int_{0}^{\infty} d k\left[\left(|A|^{2}+|B|^{2}\right)\left(2 k^{3}+\frac{k}{\eta^{2}}\right)\right. \\
& \left.+A^{*} B\left(-2 i \frac{k^{2}}{\eta}+\frac{k}{\eta^{2}}\right) e^{2 i k \eta}+A B^{*}\left(2 i \frac{k^{2}}{\eta}+\frac{k}{\eta^{2}}\right) e^{-2 i k \eta}\right],(6.4) \\
p_{\mathrm{RD}}^{\mathrm{un}-\mathrm{ren}}= & \frac{1}{8 \pi^{2} a^{4}} \int_{0}^{\infty} d k\left[\left(|A|^{2}+|B|^{2}\right)\left(\frac{2}{3} k^{3}+\frac{k}{\eta^{2}}\right)\right. \\
& \left.+A^{*} B\left(-\frac{4}{3} k^{3}-2 i \frac{k^{2}}{\eta}+\frac{k}{\eta^{2}}\right) e^{2 i k \eta}+A B^{*}\left(-\frac{4}{3} k^{3}+2 i \frac{k^{2}}{\eta}+\frac{k}{\eta^{2}}\right) e^{-2 i k \eta}\right] .
\end{aligned}
$$

\footnotetext{
${ }^{8} \mathrm{~A}$ similar term appears in a different context [34, where a time-dependent cosmological constant due to infrared effects in de Sitter space is discussed [35].

${ }^{9}$ In 36, two of the present authors discussed quadratic divergences of the Higgs boson mass term from the Wilsonian renormalization group point of view. Since they are always absorbed in the definition of critical surfaces, we argued that cutoff-dependent quadratic divergences are unphysical and should be simply subtracted. The noncovariance of quartic divergences of the cosmological constant suggests the same thing. In our opinion, power divergences should always be subtracted from the beginning, as is automatically realized in the dimensional regularization.
} 
The terms with $A^{*} B$ and $A B^{*}$ represent interference between the positive and negative frequency modes.

We now perform the subtraction of the UV divergences following the adiabatic regularization procedure. Since the potential for the wave equation vanishes in the RD period, the adiabatic wave function, or the WKB wave function, agrees with the plane-wave solution (4.8). The terms to be subtracted are given by the first line in (6.4) and (6.5) with $A=1, B=0$. Thus, the renormalized expression is obtained by simply replacing $\left(|A|^{2}+|B|^{2}\right)$ by $\left(|A|^{2}+|B|^{2}-1\right)$ in (6.4) and (6.5).

The subtraction term has been obtained for the FRW universe with the general scale factor $a$. See, for instance, eqs. (2.30) and (2.35) in ref. 32] (see also (2.10) in [33]). Substituting the present form of $a$ in (3.1), one indeed obtains the above mentioned subtraction term. Apart from that term, there are finite terms of the order $H^{4}$ (where $H$ is the Hubble parameter at each moment), which arise as a result of performing the $k$ integration in massive theory, and taking the massless limit in the end. This procedure is necessary since some of the terms in the adiabatic expansion are IR divergent, and the massless theory cannot be studied directly. These terms are important since they give the Weyl anomaly, and also contribute to the vacuum energy of pure de Sitter space. However, at late times, these terms of the order $H^{4}$ are much smaller than the total contribution that we are studying. The latter is enhanced due to the IR behavior of wave functions, as we have seen in (5.5) and (5.6). Thus, we will ignore this finite contribution of the order $H^{4}$ in this paper.

We now consider the consequences of our approximation, in which the scale factor (3.1) changes its functional forms instantaneously and its higher derivatives with respect to $\eta$ are not continuously connected at the boundaries of the stages. Due to this approximation, we have non-differentiable sharp peaks in the potential at the boundaries (see Figure 1). In this potential, the reflection coefficient decreases only with a power, $B(k) \propto k^{-2}$, as given in (4.9). Even at high $k$, over-the-barrier scattering by the sharp potential is not strongly suppressed. This is different from the general behavior for the scattering by a smooth potential, where the reflection coefficients fall off exponentially at large $k$. Namely, if wavelengths are smaller than the typical curvature radius at the peak of the potential, over-the-barrier scattering cannot occur. (See, for instance, section 52 of ref. [37.)

The power law tail of $B(k)$ for the sharp potential produces problems in the UV behavior of the $k$-integral in (6.4) and (6.5). The coefficient in the first line of (6.4) and (6.5) after the UV subtraction behaves as $\left(|A|^{2}+|B|^{2}-1\right)=$ $2|B|^{2} \propto k^{-4}$, where the unitarity relation $|A|^{2}-|B|^{2}=1$ was used. It then 
follows that the leading term $\left(|A|^{2}+|B|^{2}-1\right) k^{3} \propto k^{-1}$ gives rise to a UV logarithmic divergence. A problem also arises from the interference terms. In the IR region of $k<\eta_{2}^{-1}$, the coefficients behave as $A, B \propto k^{-2}$, as given by (4.9), and the term $A^{*} B k^{3}$ in (6.5) decreases as $1 / k$ while oscillating. The integrand $p(k)$ will be seen below in Figure 2, However, in the UV region of $k>\eta_{2}^{-1}$, the coefficients behave as $A \propto 1$ and $B \propto k^{-2}$, and the term $A^{*} B k^{3}$ grows as a linear function of $k$ though it oscillates.

These problems are caused by our setting where the scale factors are not sufficiently smoothly connected at the boundaries of the stages. The subtraction term obtained by the DeWitt-Schwinger expansion or adiabatic regularization, which is based on the adiabatic expansion of the background geometry, could not cancel all the UV divergences in such cases. Since modes with infinitesimally small wavelengths are affected by the sharp potential at the boundary, the UV behaviors at later times become dependent on the past history and cannot be controlled only by the local quantities.

We assume that the scale factors in the realistic settings are smoothly connected, so that the reflection coefficients decay quickly when $k \gtrsim \eta_{2}^{-1}$. To take this behavior into account, we will introduce a mask function $f_{2}(k)$, which takes a value close to 1 for $k \lesssim \eta_{2}^{-1}$, and falls off rapidly for $k \gtrsim \eta_{2}^{-1}$. The scattering coefficients have to satisfy the unitarity relation $|A|^{2}-|B|^{2}=1$. The simplest way to apply a mask function, $f_{2}(k)$, that is consistent with this relation would be to make the following replacements in (4.9):

$$
\frac{1}{\eta_{1}} \rightarrow \frac{f_{2}(k)}{\eta_{1}}, \frac{1}{\eta_{1}^{2}} \rightarrow \frac{f_{2}(k)^{2}}{\eta_{1}^{2}}
$$

In the actual analysis, we will take

$$
f_{2}(k)=\left\{\begin{array}{ll}
1 & \left(k \leq \eta_{2}^{-1}\right) \\
0 & \left(k>\eta_{2}^{-1}\right)
\end{array} .\right.
$$

Note that, after the subtraction of UV divergences, all the terms in $\left(|A|^{2}+\right.$ $\left.|B|^{2}-1\right), A^{*} B$, and $A B^{*}$ contain a factor of $1 / \eta_{1}^{n}(n \geq 1)$. Hence, owing to $f_{2}^{n}=f_{2}$, the effect of introducing the mask function is to set the upper bound of the $k$ integration at $1 / \eta_{2}$. As we will see at the end of Section 7.1, the final results do not depend much on the explicit form of the mask function 10

To summarize, after the subtraction of the UV divergences and the smoothing of the potential, $\rho_{\mathrm{RD}}$ and $p_{\mathrm{RD}}$ are given by (6.4) and (6.5) with the following

\footnotetext{
10 The UV divergence caused by the sharp changes of the background has been noticed also in [14]. The authors of [14] introduced a regulator function, which decays exponentially at large $k$, to model the behavior of a smooth transition. Our masking procedure is very similar to this in spirit, and will give essentially the same result.
} 
replacements:

$$
\begin{aligned}
\left(|A|^{2}+|B|^{2}\right) & \longrightarrow\left(|A|^{2}+|B|^{2}-1\right) \\
\int_{0}^{\infty} d k & \longrightarrow \int_{0}^{\eta_{2}^{-1}} d k
\end{aligned}
$$

\subsection{MD period}

In the MD period, by substituting the wave function (4.6) into (5.2) and (5.4), one obtains the energy and pressure densities:

$$
\begin{aligned}
\rho_{\mathrm{MD}}^{\mathrm{un}-\mathrm{ren}}= & \frac{1}{8 \pi^{2} a^{4}} \int_{0}^{\infty} d k\left[\left(|C|^{2}+|D|^{2}\right)\left(2 k^{3}+4 \frac{k}{\eta^{2}}+9 \frac{1}{k \eta^{4}}\right)\right. \\
& +C^{*} D 2 k^{3}\left(7(k \eta)^{-2}-\frac{9}{2}(k \eta)^{-4}+\left(-2(k \eta)^{-1}+9(k \eta)^{-3}\right) i\right) e^{2 i k \eta} \\
& +C D^{*} 2 k^{3}\left(7(k \eta)^{-2}-\frac{9}{2}(k \eta)^{-4}-\left(-2(k \eta)^{-1}+9(k \eta)^{-3}\right) i\right) e^{-2 i k \eta],} \\
p_{\mathrm{MD}}^{\mathrm{un}-\mathrm{ren}=} & \frac{1}{8 \pi^{2} a^{4}} \int_{0}^{\infty} d k\left[\left(|C|^{2}+|D|^{2}\right)\left(\frac{2}{3} k^{3}+\frac{8}{3} \frac{k}{\eta^{2}}+9 \frac{1}{k \eta^{4}}\right)\right. \\
& +C^{*} D 2 k^{3}\left(-\frac{2}{3}+\frac{23}{3}(k \eta)^{-2}-\frac{9}{2}(k \eta)^{-4}+\left(-\frac{10}{3}(k \eta)^{-1}+9(k \eta)^{-3}\right) i\right) e^{2 i k \eta} \\
& +C D^{*} 2 k^{3}\left(-\frac{2}{3}+\frac{23}{3}(k \eta)^{-2}-\frac{9}{2}(k \eta)^{-4}-\left(-\frac{10}{3}(k \eta)^{-1}+9(k \eta)^{-3}\right) i\right) e^{-2 i k \eta]} .
\end{aligned}
$$

The subtraction of UV divergences is similarly performed by replacing $\left(|C|^{2}+\right.$ $\left.|D|^{2}\right)$ by $\left(|C|^{2}+|D|^{2}-1\right)$ in (6.9) and (6.10). In the MD period, the adiabatic expression is given by the first line in (6.9) and (6.10) with $C=1, D=0$. One can see this by expanding the wave function (4.7) in $1 /(k \eta)$, or by substituting our scale factor $a(\eta)$ into the general expression in [32, 33]. As we mentioned in the previous subsection, we ignore the finite terms of the order $H^{4}$ arising from renormalization, since they are smaller than the terms of interest at late times.

For the masking procedure, in addition to the replacement of (6.6) with (6.7) in (4.9), we can also make the replacement

$$
\frac{1}{\eta_{4}} \rightarrow \frac{f_{4}(k)}{\eta_{4}}, \frac{1}{\eta_{4}^{2}} \rightarrow \frac{f_{4}(k)^{2}}{\eta_{4}^{2}}
$$

with

$$
f_{4}(k)= \begin{cases}1 & \left(k \leq \eta_{4}^{-1}\right) \\ 0 & \left(k>\eta_{4}^{-1}\right)\end{cases}
$$


in (4.10). The latter is introduced for smoothing the potential peak at the matter-radiation equality $\eta=\eta_{4}$. Then the scattering amplitudes $C(k)$ and $D(k)$ reduce to $A(k)$ and $B(k)$ for $k>\eta_{4}^{-1}$. They further become $C(k)=1$ and $D(k)=0$ for $k>\eta_{2}^{-1}$, which truncates the $k$-integral at $k=\eta_{2}^{-1}$, as in the $\mathrm{RD}$ period case. However, the results do not depend much on whether or not we replace $C$ and $D$ by $A$ and $B$ in the region $k \in\left[\eta_{4}^{-1}, \eta_{2}^{-1}\right]$. Since the leading contributions to the scattering coefficients $C$ and $D$ for the modes $k \in\left[\eta_{4}^{-1}, \eta_{2}^{-1}\right]$ come from the waves that are scattered by the potential in the inflation period but pass through the potential in the MD period, over-the-barrier scatterings by the potential in the MD period give only sub-leading contributions and do not affect the results much. We therefore will not perform the replacement of $C$ and $D$ by $A$ and $B$ in the region $k \in\left[\eta_{4}^{-1}, \eta_{2}^{-1}\right]$.

To summarize, our formula in the MD period is as follows. $\rho_{\mathrm{MD}}$ and $p_{\mathrm{MD}}$ are given by (6.9) and (6.10), with the replacement of $\left(|C|^{2}+|D|^{2}\right)$ by $\left(|C|^{2}+|D|^{2}-\right.$ $1)$, modifying the subtraction term in the IR region properly, and introducing an upper bound of the $k$ integration at $\eta_{2}^{-1}$.

\section{$7 \quad$ Time evolution of energy and pressure densities}

We are now ready to study the time evolution of the energy and pressure densities in the cosmic history after the inflation period.

\subsection{RD period}

In the RD period, the energy and pressure densities are given by (6.4) and (6.5) with modifications by the UV subtraction and masking procedures addressed at the end of Section 6.1. With (4.9) plugged in, they become

$$
\begin{aligned}
\rho_{\mathrm{RD}}= & \frac{1}{8 \pi^{2} a^{4} \eta_{1}^{4}} \int_{0}^{\eta_{2}^{-1}} \frac{d k}{k}\left[1-(k \eta)^{-1}\left(s-2 k \eta_{1} c-2\left(k \eta_{1}\right)^{2} s\right)\right. \\
+ & \left.(k \eta)^{-2}\left(\frac{1}{2}-\frac{1}{2} c-k \eta_{1} s+\left(k \eta_{1}\right)^{2} c\right)\right] \\
p_{\mathrm{RD}}= & \frac{1}{8 \pi^{2} a^{4} \eta_{1}^{4}} \int_{0}^{\eta_{2}^{-1}} \frac{d k}{k}\left[\frac{1}{3}+\frac{2}{3} c+\frac{4}{3} k \eta_{1} s-\frac{4}{3}\left(k \eta_{1}\right)^{2} c\right. \\
& -(k \eta)^{-1}\left(s-2 k \eta_{1} c-2\left(k \eta_{1}\right)^{2} s\right) \\
& \left.+(k \eta)^{-2}\left(\frac{1}{2}-\frac{1}{2} c-k \eta_{1} s+\left(k \eta_{1}\right)^{2} c\right)\right]
\end{aligned}
$$


where $s=\sin \left[2 k\left(\eta-\eta_{2}\right)\right]$ and $c=\cos \left[2 k\left(\eta-\eta_{2}\right)\right]$. The prefactor in (7.1) and $(7.2)$ is rewritten as

$$
\frac{1}{8 \pi^{2} a^{4} \eta_{1}^{4}}=\frac{1}{8 \pi^{2} a^{4}}\left(a_{\mathrm{Inf}}\left(\eta_{1}\right) H_{I}\right)^{4}=\frac{1}{8 \pi^{2}} H_{I}^{4}\left(\frac{H}{H_{I}}\right)^{2}=\frac{1}{8 \pi^{2}}\left(H_{I} H\right)^{2},
$$

where we have used (3.1) for the first equality, and the relation $H^{2} \propto a^{-4}$ in the RD period for the second equality. Here, $H_{I}$ and $H$ are the Hubble parameters at the inflation period and at the time of interest. As we have noticed before, $\rho$ and $p$ are enhanced to the order of (17.3). The modes with $k<\eta_{2}^{-1}$ are enhanced due to the non-adiabatic evolution in the inflation period.

The integrands (without the prefactor $1 / 8 \pi^{2} a^{4} \eta_{1}^{4}$ ) of (17.1) and (7.2) are shown in Figure 2, They oscillate and decrease as $k$ increases. The oscillation period is $\Delta k=\pi / \eta$. Under the conditions of $\eta \gg \eta_{2}=\left|\eta_{1}\right|$ and $k \ll 1 / \eta_{2}$, the height of each peak increases in proportion to $\eta$. This behavior can easily be seen from the fact that under the above conditions, $\eta_{2}=\left|\eta_{1}\right|$ can be dropped from the integrands without the prefactor, and they are invariant under $k \rightarrow \lambda k$ and $\eta \rightarrow \eta / \lambda$. Thus, the integration over each peak remains almost constant irrespective of $\eta$.

Time evolution of $\rho$ and $p$, besides the time dependence of the prefactor (7.3), comes from the finite integration range of the $k$-integral, $k \in\left[0, \eta_{2}^{-1}\right]$. Since the oscillation period is $\Delta k=\pi / \eta$, the number of peaks within the interval is given by $\eta /\left(\pi \eta_{2}\right)$, which grows with time. For small values of $\eta / \eta_{2}$, i.e., just after inflation, the integration is mainly contributed to by the first few peaks of the integrands in the IR regions. On the contrary, for the later stage with large values of $\eta / \eta_{2}$, the integration is dominated by the UV tail of the integrands.

Before discussing the time evolution in more detail, we first investigate the analytical behaviors of the integrals in the IR and UV regions separately. In the IR region, the integrals of (17.1) and (7.2) seem to diverge, but, as we saw before, cancellations among various terms occur and they become IR finite. Indeed, (7.1) and (7.2) are estimated in the IR region as

$$
\begin{aligned}
\rho_{\mathrm{RD}}^{\mathrm{IR}} & =\frac{1}{8 \pi^{2} a^{4} \eta_{1}^{4}} \int_{0} d k\left[\left(1-\left(\frac{\eta_{2}}{\eta}\right)^{4}\right) \eta^{2} k+\mathcal{O}\left(k^{3}\right)\right] \\
p_{\mathrm{RD}}^{\mathrm{IR}} & =\frac{1}{8 \pi^{2} a^{4} \eta_{1}^{4}} \int_{0} d k\left[-\frac{1}{3}\left(1+3\left(\frac{\eta_{2}}{\eta}\right)^{4}\right) \eta^{2} k+\mathcal{O}\left(k^{3}\right)\right] .
\end{aligned}
$$

The second term in the parenthesis, which is proportional to $\left(\eta_{2} / \eta\right)^{4}$, comes from the subtraction term. The leading terms reproduce eqs. (5.5) and (5.6) by using (7.3) and the relation $a \eta=H^{-1}$ in the RD period. Therefore, the 


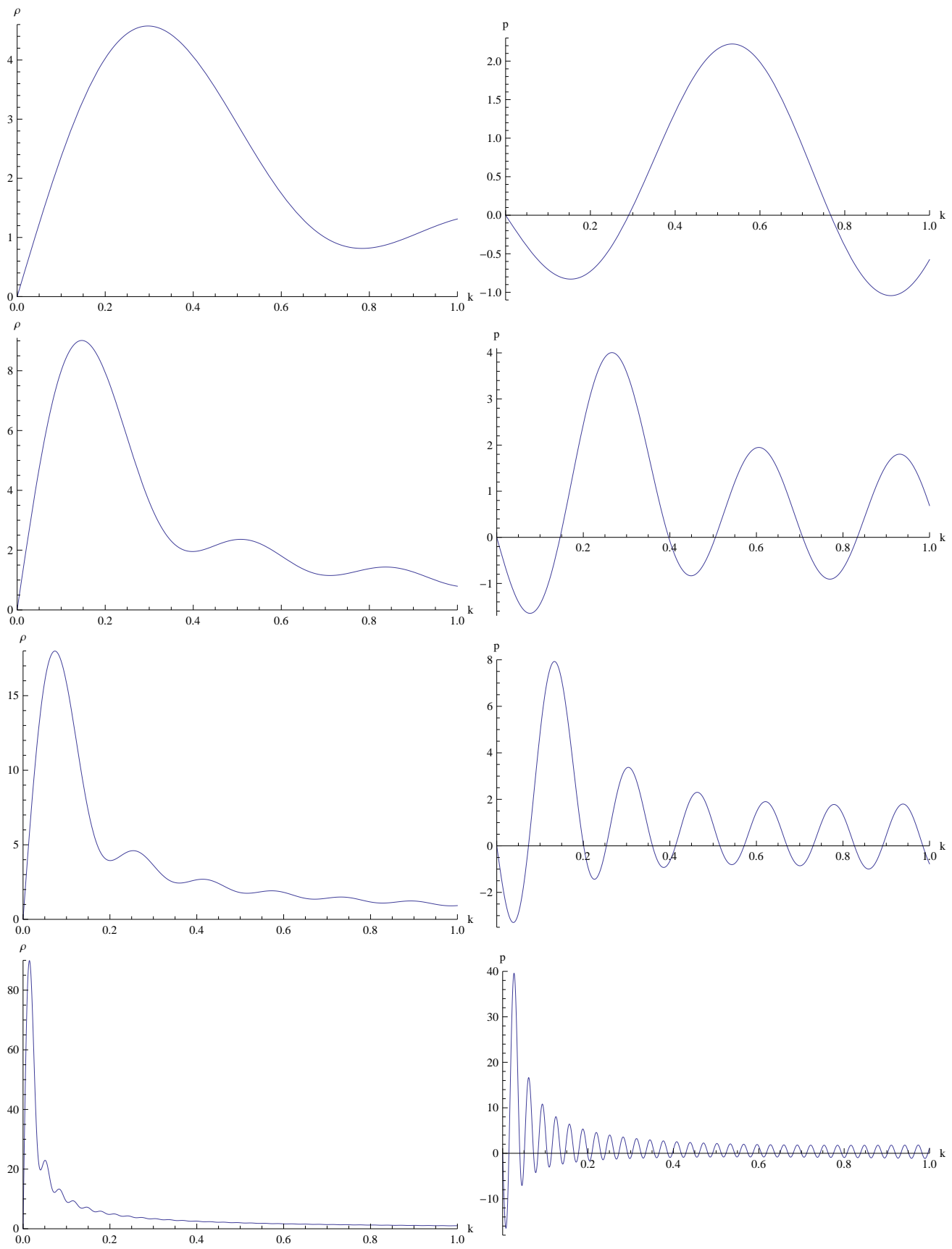

Figure 2: Integrands $\rho(k)$ of (7.1) and $p(k)$ of (7.2), without the prefactor $1 / 8 \pi^{2} a^{4} \eta_{1}^{4}$, are shown over the integration interval $k \in\left[0, \eta_{2}^{-1}\right]$. The parameters are taken to be $\eta_{2}=-\eta_{1}=1$ and $\eta=5,10,20,100$ from the top to the bottom figures. 
contribution to the integral (7.1) from the IR region below the first peak in Figure 2 is evaluated as

$$
\rho_{\mathrm{RD}}^{\mathrm{IR}} \sim \frac{1}{8 \pi^{2} a^{4} \eta_{1}^{4}} \int_{0}^{\pi / 2 \eta} d k \eta^{2} k=\frac{1}{8 \pi^{2}}\left(H_{I} H\right)^{2} \eta^{2} \frac{1}{2}\left(\frac{\pi}{2 \eta}\right)^{2}=\frac{1}{64}\left(H_{I} H\right)^{2} .
$$

The first peak is located approximately at $\pi / 2 \eta$. In the second equality, (7.3) was used. If we further perform the integration up to the first minimum of $\rho$, it becomes almost doubled:

$$
\rho_{\mathrm{RD}}^{\mathrm{IRpeak}} \sim\left(H_{I} H\right)^{2} / 32 .
$$

For $k<\pi /(4 \eta)$, the pressure density $p$ is approximated by (7.5) and behaves as $p \sim-\rho / 3$. However, as shown in Figure 2, the integrand of $p$ changes its sign from negative to positive around $k=\pi /(2 \eta)$, and the equation of state $w=\rho / p$ gradually changes.

On the other hand, in the UV region with $k \eta \gg 1$, the energy and pressure densities are approximately given by the first term in the square bracket of (7.1) and (7.2) 11 We then obtain 12

$$
\begin{aligned}
& \rho_{\mathrm{RD}}^{\mathrm{UV}} \simeq \frac{1}{8 \pi^{2} a^{4} \eta_{1}^{4}} \int_{\sim \eta^{-1}}^{\eta_{2}^{-1}} \frac{d k}{k}=\frac{1}{8 \pi^{2}}\left(H_{I} H\right)^{2} \ln \left(\frac{\eta}{\eta_{2}}\right), \\
& p_{\mathrm{RD}}^{\mathrm{UV}} \simeq \frac{1}{8 \pi^{2} a^{4} \eta_{1}^{4}} \int_{\sim \eta^{-1}}^{\eta_{2}^{-1}} \frac{d k}{k} \frac{1}{3}=\frac{1}{3} \rho_{\mathrm{RD}}^{\mathrm{UV}},
\end{aligned}
$$

where (7.3) is used in the second equality in (7.8). By comparing (7.7) and (7.8), we find that the UV tail contribution becomes larger than the first IR peak contribution when $\eta / \eta_{2}>e^{\pi^{2} / 4} \sim 12$.

We now show the numerical evaluations of the time evolution of $\rho$ and $p$ in the RD period. Figure 3 shows the early time behavior for $1<\eta / \eta_{2}<10$. The position of the first minimum of $\rho(k)$ is at $k=\pi / \eta$ and the integration is taken over the interval $k \in\left[0, \eta_{2}^{-1}\right]$. Hence, for $\eta \sim \pi \eta_{2}, \rho$ is approximated

\footnotetext{
${ }^{11}$ The other terms in (7.1) may give similar contributions to (7.8), but, compared to the logarithmic factor $\ln \left(\eta / \eta_{2}\right)$, they are at most of the order of one and thus negligible. For instance, the second term in (7.1) becomes $\int_{\eta^{-1}}^{\eta_{2}^{-1}} d k k^{-2} \eta^{-1} \sin (2 k \eta) \sim 1$, since only the region around $k \sim \eta^{-1}$ contributes. The third term in the first parenthesis, an oscillating but non-decreasing function of $k$, becomes $\int_{\eta^{-1}}^{\eta_{2}^{-1}} d k \eta^{-1} \eta_{1}^{2} \sin (2 k \eta) \sim\left(\eta_{1} / \eta\right)^{2} \ll 1$. The first term in the second parenthesis, which is not oscillating, becomes $\int_{\eta^{-1}}^{\eta_{2}^{-1}} d k k^{-3} \eta^{-2} \sim 1$. The most worrisome term is the fourth term in (7.2), which is oscillating but increasing. As we mentioned before, this is an artifact of the potential with a sharp peak and cured by introducing the mask function. This term gives $\int_{\eta^{-1}}^{\eta_{2}^{-1}} d k k \eta_{1}^{2} \cos (2 k \eta) \sim\left|\eta_{1}\right| / \eta \ll 1$, since only the region around $k \sim \eta_{2}^{-1}=\left|\eta_{1}\right|^{-1}$ with the width $\eta^{-1}$ contributes.

12 These expressions agree with the ones obtained in [14].
} 


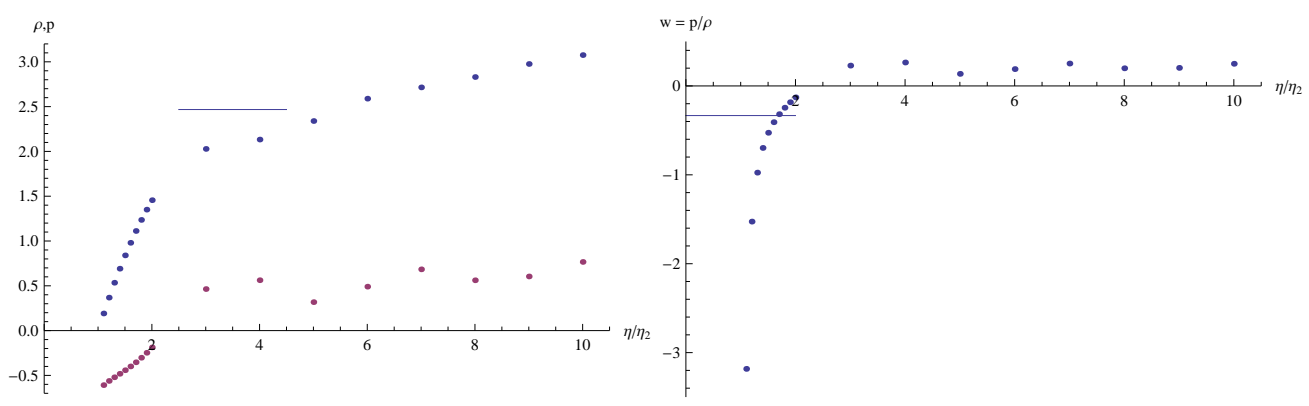

Figure 3: Early time behavior of the time evolution of $\rho$ and $p$ in the RD period. We take $1<\eta / \eta_{2}<10$, i.e., the time $\eta$ is close to the big bang, $\eta_{2}$. $\rho$ gradually increases while $p$ changes its sign from negative to positive as it oscillates. The very early time behavior below $\eta / \eta_{2}<1.5$ is an artifact of the UV subtraction and cannot be taken at face value. (Left) The upper and lower data represent $\rho$ and $p$, i.e., (7.1) and (7.2) without the prefactor $1 / 8 \pi^{2} a^{4} \eta_{1}^{4}$. The line represents the first IR peak contribution (7.7). (Right) The equation of state $w=p / \rho$ is shown. The line represents $w=-1 / 3$, a value given by (5.5) and (5.6) .

by the first peak contribution (17.7), which is depicted by the line in the figure. As $\eta / \eta_{2}$ increases, the energy density $\rho$ gradually grows by taking the second and the following peak contributions. The behavior of $p$ is more complicated. The integrand $p(k)$ is negative for $k<\pi /(2 \eta)$ and changes its sign around $k=$ $\pi /(2 \eta)$. Hence, the $k$-integral of $p$ is negative until $\eta \sim 2 \eta_{2}$, and then becomes positive. Reflecting the oscillating behavior of the integrand in Figure 2, $p$ gradually increases and oscillates in Figure 3. The data in Figure 3 for $\eta / \eta_{2} \lesssim$ 1.5 cannot be taken seriously 13 , since the spacetime curvature is not small in this region, and the corrections due to finite renormalization terms from the UV subtraction, which were discussed in the third paragraph of Section 6.1, cannot be ignored. Figure 4 shows the late time behaviors of (7.1) and (7.2) for $10<\eta / \eta_{2}<10^{30}$. As expected, the results agree well with Eqs. (7.8) and (7.9).

Finally, we will see that the integrals of $\rho$ and $p$ are not sensitive to the details of the masking function introduced to express the smooth potential. For example, if we change the upper bound of the $k$-integral from $1 / \eta_{2}$ to $r / \eta_{2}$, the logarithmic factor in (7.8) is changed from $\ln \left(\eta / \eta_{2}\right)$ to $\left(\ln \left(\eta / \eta_{2}\right)+\ln r\right)$. The difference is very small as long as $r \ll\left(\eta / \eta_{2}\right)$.

\footnotetext{
${ }^{13}$ For $\eta / \eta_{2}<2$, the contribution to $p$ is mainly given by the first negative peak, and $w$ becomes negative. As seen below (5.5) and (5.6), $w \rightarrow-1 / 3$ is expected for $\eta / \eta_{2} \rightarrow 1$. However, $w$ takes even smaller values because the second term in (7.4) and (7.5), which comes from the subtraction term, becomes the same order as the first term.
} 


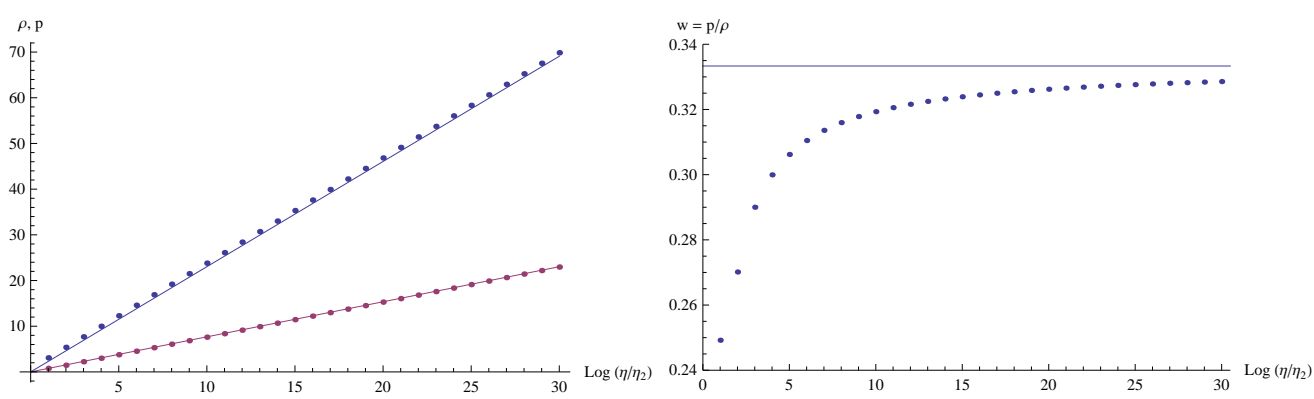

Figure 4: Late time behavior of the time evolution of $\rho$ and $p$ in the RD period. We take $10<\eta / \eta_{2}<10^{30}$, i.e., the time $\eta$ is far from the big bang, $\eta_{2}$. (Left) The upper and lower data represent $\rho$ and $p$, i.e., (7.1) and (7.2) without the prefactor $1 / 8 \pi^{2} a^{4} \eta_{1}^{4}$. The lines represent the analytical approximations (7.8) and (7.9), which agree well with the numerical evaluations. (Right) The equation of state $w=p / \rho$ is shown. The line represents $w=1 / 3$, a value predicted by (17.8) and (7.9).

\subsection{MD period}

In the MD period, the energy and pressure densities are given by the formula (6.9) and (6.10), modified by the subtraction and masking procedures explained at the end of Section 6.2 ,

Figure 5 shows the integrands in the IR region $k \in\left[0,0.1 \eta_{2}^{-1}\right]$, where $\eta_{2}^{-1}$ is the upper bound of the $k$-integration. The top figures correspond to the time when the MD period begins at $\eta=\eta_{4}$. They indeed agree with the figures when the RD period ends at $\eta=\eta_{3}$, which are shown by the bottom figures in Figure 2. As can be seen from (4.10), the oscillation period is given by $\Delta k=\pi /\left(\eta-\eta_{4}+\eta_{3}\right)$, and decreases as $\eta$ increases. A notable feature in Figure 5 is that the relative height of the peaks in the IR region to those in the $\mathrm{UV}$ region becomes larger as $\eta$ evolves.

Before discussing the time evolution, we first investigate the integrals in the IR and UV regions separately. For the IR region with $k<\eta_{4}^{-1}$, the coefficients of the wave function $C$ and $D$ are approximated by (B.4 and (B.5). Substituting them into (6.9) and (6.10), we obtain

$$
\begin{aligned}
\rho_{\mathrm{MD}}^{\mathrm{IR}} \simeq & \frac{1}{8 \pi^{2} a^{4} \eta_{1}^{4}}\left(\frac{\eta}{\eta_{4}}\right)^{2} \int_{0} d k \frac{9}{8} k^{-3} \eta^{-2}\left[2+4(k \eta)^{-2}+9(k \eta)^{-4}\right. \\
& +\left(14(k \eta)^{-2}-9(k \eta)^{-4}\right) \cos (2 k \eta) \\
& \left.+\left(4(k \eta)^{-1}-18(k \eta)^{-3}\right) \sin (2 k \eta)\right]
\end{aligned}
$$



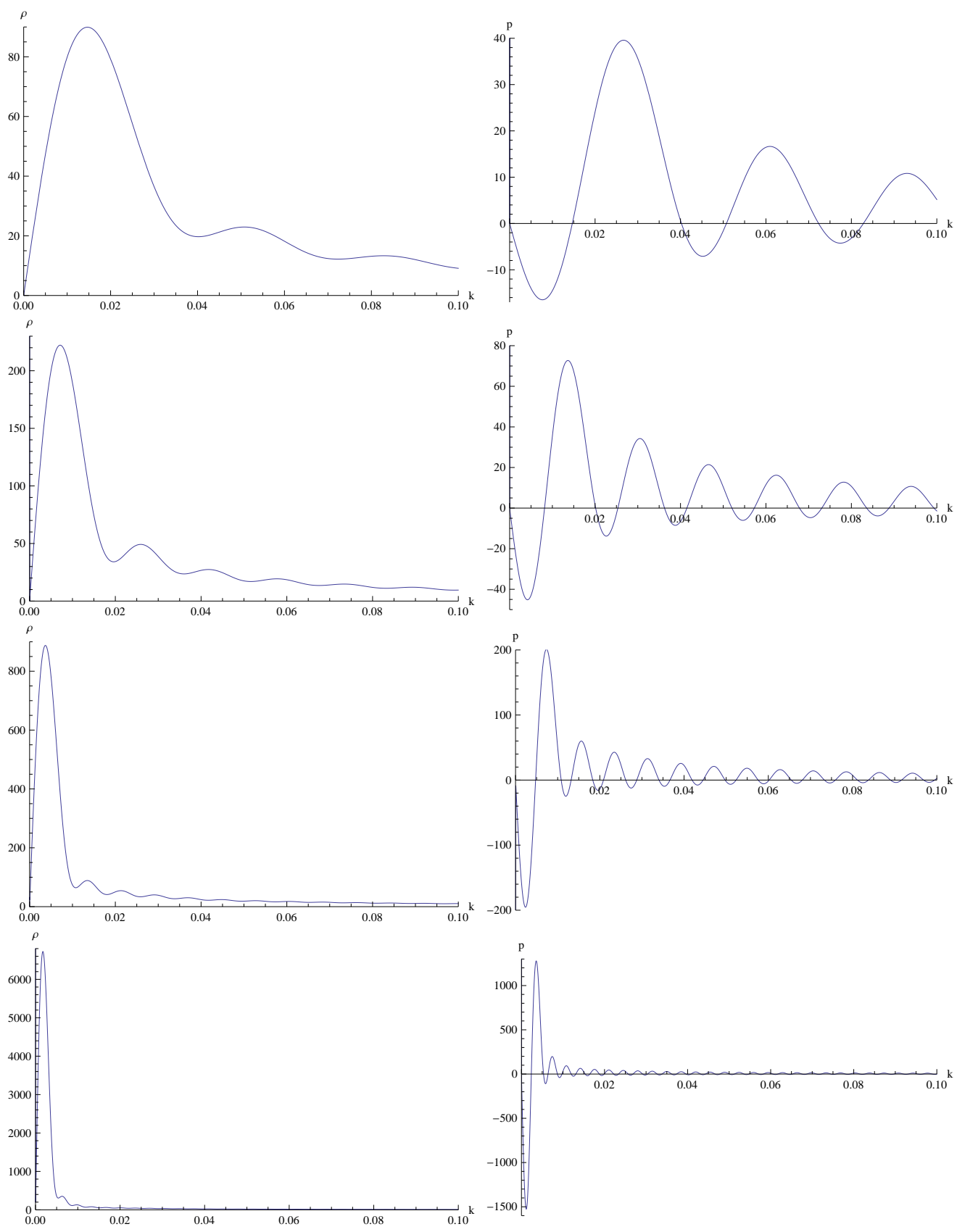

Figure 5: Integrands $\rho(k)$ and $p(k)$ in the MD period. (6.9) and (6.10), multiplied by $8 \pi^{2} a^{4} \eta_{1}^{4}$, are depicted for $k \in\left[0,0.1 \eta_{2}^{-1}\right]$. The parameters are taken to be $\eta_{2}=-\eta_{1}=1, \eta_{4}=2 \eta_{3}=200$, and $\eta=200,300,500,1000$ from the top to the bottom figures. The top figures correspond to the time when the MD period starts, which is the same as the bottom figures of Figure 2, corresponding to the time when the RD period ends. 


$$
\begin{aligned}
p_{\mathrm{MD}}^{\mathrm{IR}} \simeq & \frac{1}{8 \pi^{2} a^{4} \eta_{1}^{4}}\left(\frac{\eta}{\eta_{4}}\right)^{2} \int_{0} d k \frac{3}{8} k^{-3} \eta^{-2}\left[2+8(k \eta)^{-2}+27(k \eta)^{-4}\right. \\
& +\left(-4+46(k \eta)^{-2}-27(k \eta)^{-4}\right) \cos (2 k \eta) \\
& \left.+\left(20(k \eta)^{-1}-54(k \eta)^{-3}\right) \sin (2 k \eta)\right] .
\end{aligned}
$$

Note that an extra prefactor $\left(\eta / \eta_{4}\right)^{2}$ appears. This is the reason for the special enhancement of the peaks in the IR region. By using the relation $a \propto \eta^{2}$ in the MD period, it is written as

$$
\left(\frac{\eta}{\eta_{4}}\right)^{2}=\frac{a}{a_{\mathrm{eq}}}
$$

where eq stands for the matter-radiation equality. However, unlike (7.3) in the $\mathrm{RD}$ period, the following relation holds in the MD period:

$$
\frac{1}{8 \pi^{2} a^{4} \eta_{1}^{4}}=\frac{1}{8 \pi^{2}}\left(H_{I} H\right)^{2}\left(\frac{a_{\mathrm{eq}}}{a}\right)
$$

Here, we used

$$
\left(\frac{a_{\mathrm{Inf}}\left(\eta_{1}\right)}{a}\right)^{4}=\left(\frac{a_{\mathrm{Inf}}\left(\eta_{1}\right)}{a_{\mathrm{eq}}}\right)^{4}\left(\frac{a_{\mathrm{eq}}}{a}\right)^{3}\left(\frac{a_{\mathrm{eq}}}{a}\right)=\left(\frac{H}{H_{I}}\right)^{2}\left(\frac{a_{\mathrm{eq}}}{a}\right),
$$

which follows the relations $H^{2} \propto a^{-4}, a^{-3}$ in the RD and MD periods, respectively. Then the prefactor including the extra factor (7.12) becomes

$$
\frac{1}{8 \pi^{2} a^{4} \eta_{1}^{4}}\left(\frac{\eta}{\eta_{4}}\right)^{2}=\frac{1}{8 \pi^{2}}\left(H_{I} H\right)^{2} .
$$

Hence, in the unit of $\left(H_{I} H\right)^{2}$, the heights of the IR peaks are the same as in the RD period. The heights of UV peaks, as we see below in (7.20), do not have such an extra enhancement factor, and thus are reduced by the factor $a_{\text {eq }} / a$.

If we further expand $\cos (2 k \eta)$ and $\sin (2 k \eta)$ in Eqs. (7.10) and (7.11), we obtain IR finite integrals:

$$
\begin{aligned}
\rho_{\mathrm{MD}}^{\mathrm{IR}} & =\frac{1}{8 \pi^{2} a^{4} \eta_{1}^{4}}\left(\frac{\eta}{\eta_{4}}\right)^{2} \int_{0} d k\left[\frac{\eta^{2}}{4} k+\mathcal{O}\left(k^{2}\right)\right], \\
p_{\mathrm{MD}}^{\mathrm{IR}} & =\frac{1}{8 \pi^{2} a^{4} \eta_{1}^{4}}\left(\frac{\eta}{\eta_{4}}\right)^{2} \int_{0} d k\left[-\frac{\eta^{2}}{12} k+\mathcal{O}\left(k^{2}\right)\right] .
\end{aligned}
$$

They indeed reproduce (5.5) and (5.6) by using (17.15) and the relation $a \eta=$ $2 H^{-1}$ in the MD period.

We now perform the $k$-integration of (17.10) and (7.11) over the interval $k \in\left[0, \eta_{4}^{-1}\right]$, in which the approximation is valid. The integrands are shown in Figure 6. They damp quickly as $k^{-3}$, and take negligibly small values for 


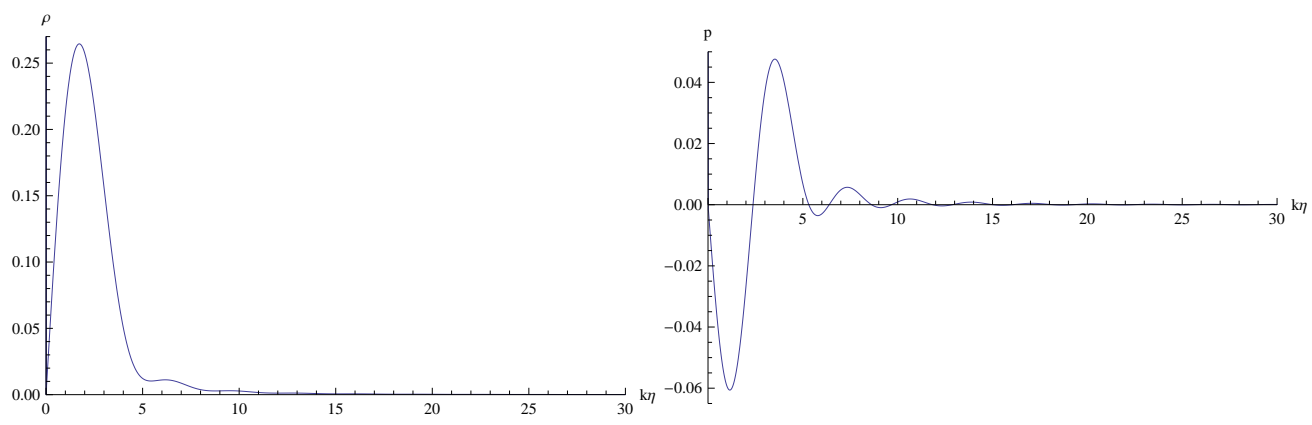

Figure 6: Integrands of (7.10) and (7.11), without the prefactor $\left(8 \pi^{2} a^{4} \eta_{1}^{4}\right)^{-1}\left(\eta / \eta_{4}\right)^{2}$, are plotted as a function of $k \eta$.

$k \gtrsim 5 \eta^{-1}$. Hence, for $\eta \gtrsim 5 \eta_{4}$, the integral over $k \in\left[0, \eta_{4}^{-1}\right]$ becomes almost constant and equal to that over $k \in[0, \infty]$ :

$$
\begin{aligned}
& \rho_{\mathrm{MD}}^{\mathrm{IR}} \simeq \frac{3}{4} \frac{1}{8 \pi^{2}}\left(H_{I} H\right)^{2}, \\
& p_{\mathrm{MD}}^{\mathrm{IR}} \simeq 0 .
\end{aligned}
$$

The equation of state becomes $w^{\mathrm{IR}}=p^{\mathrm{IR}} / \rho^{\mathrm{IR}}=0$ in the IR region.

We next examine the behaviors of the UV region. For $k>\eta_{4}^{-1}$, the coefficients $C$ and $D$ in the wave function become close to $A$ and $B$ since those waves do not scatter in the MD period. Furthermore, the difference of the integrands (6.9) and (6.10) from those in the RD period in (6.4) and (6.5) are higher orders of $1 /(k \eta)$ and negligible. Then one can perform the same analysis mentioned below (7.8) and (7.9), and obtain

$$
\begin{aligned}
\rho_{\mathrm{MD}}^{\mathrm{UV}} & \simeq \frac{1}{8 \pi^{2} a^{4} \eta_{1}^{4}} \int_{\eta_{4}^{-1}}^{\eta_{2}^{-1}} \frac{d k}{k}=\frac{1}{8 \pi^{2}}\left(H_{I} H\right)^{2}\left(\frac{a_{\mathrm{eq}}}{a}\right) \ln \left(\frac{\eta_{4}}{\eta_{2}}\right) \\
p_{\mathrm{MD}}^{\mathrm{UV}} & \simeq \frac{1}{8 \pi^{2} a^{4} \eta_{1}^{4}} \int_{\eta_{4}^{-1}}^{\eta_{2}^{-1}} \frac{d k}{k} \frac{1}{3}=\frac{1}{3} \rho_{\mathrm{MD}}^{\mathrm{UV}}
\end{aligned}
$$

where (7.13) has been used in the second equality. The UV contribution gives the equation of state $w^{\mathrm{UV}}=p^{\mathrm{UV}} / \rho^{\mathrm{UV}}=1 / 3$. As we will see shortly, this does not mean $w$ approaches $w^{\mathrm{UV}}=1 / 3$ as $\eta \rightarrow \infty$.

We now show the time evolution of $\rho$ and $p$ in the MD period. The sum of the IR contribution (7.18) and the UV contribution (7.20) gives the total amount of $\rho$. Similarly, the sum of (7.19) and (7.21) gives $p$. They are depicted in Figure 7 . When the MD period begins, the UV contribution (7.20) dominates the IR contribution (7.18) because of the extra factor $\ln \left(\eta_{4} / \eta_{2}\right) \lesssim \ln \left(3.5 \cdot 10^{25}\right) \sim$ 59 in the UV contribution. Here, the explicit values (3.17) and (3.19) are used. The equation of state is then $w \simeq 1 / 3$, which agrees with the late time 


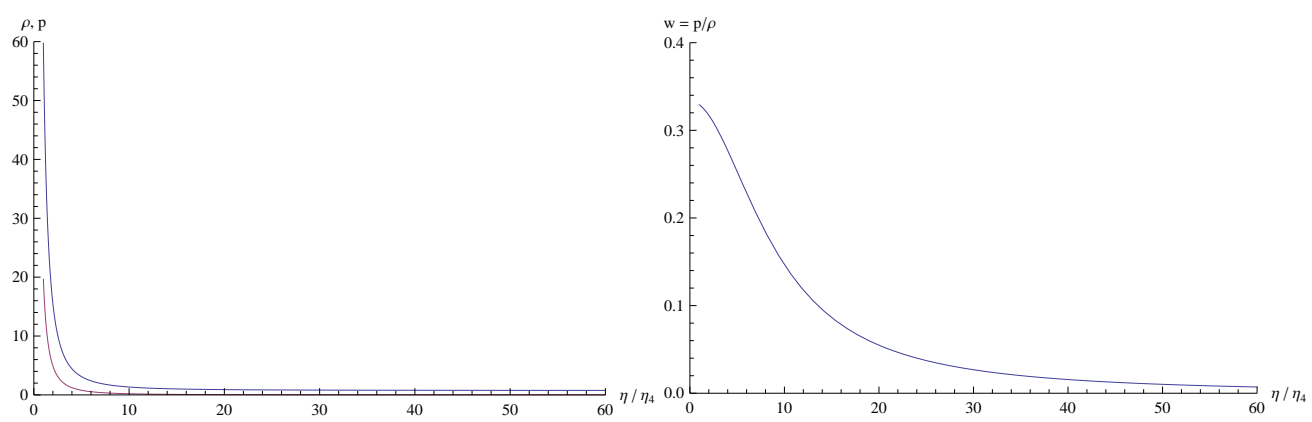

Figure 7: Time evolutions in the MD period. (Left) The upper and lower curves show the time evolutions of $\rho$ and $p$, in the unit of $\left(H_{I} H\right)^{2} /(8 \pi)^{2}$. (Right) The time evolution of the equation of state $w=p / \rho$ is shown. The parameter is taken to be $\ln \left(\eta_{4} / \eta_{2}\right)=59$.

behavior in the RD period. This should be the case since $\rho$ and $p$ are connected continuously. As time passes, however, another factor, $a_{\mathrm{eq}} / a=\left(\eta_{4} / \eta\right)^{2}$, in (7.20) reduces the UV contribution, and eventually the IR contribution (7.18) dominates over (7.20). Since the IR contribution for the pressure density $p$ vanishes, as shown in (7.19), $p$ evolves according to (7.21) , and the equation of state approaches $w \simeq 0$.

To summarize, the equation of state for the energy density of a quantum field starts from $w \simeq-1$ in the inflation era, turns into $w \simeq 1 / 3$ in the $\mathrm{RD}$ period, and then into $w \simeq 0$ in the MD period. It is interesting that the equation of state of the induced energy density coincides with that of the background geometry. This is consistent with the fact that $\rho$ and $p$ behave as $\left(H_{I} H\right)^{2}$, and thus have time dependence of $H^{2}$.

Finally, the present energy and pressure densities at $\eta=\eta_{0}$ become 14

$$
\begin{aligned}
& \rho_{0} \simeq \rho_{\mathrm{MD}}^{\mathrm{IR}} \simeq \frac{3}{32 \pi^{2}}\left(H_{I} H_{0}\right)^{2} \\
& p_{0} \simeq p_{\mathrm{MD}}^{\mathrm{UV}} \simeq \frac{1}{24 \pi^{2}}\left(\frac{a_{\mathrm{eq}}}{a_{0}}\right) \ln \left(\frac{\eta_{4}}{\eta_{2}}\right)\left(H_{I} H_{0}\right)^{2}<7.0 \times 10^{-5}\left(H_{I} H_{0}\right)^{2},
\end{aligned}
$$

where $a_{0} / a_{\text {eq }} \sim 3.4 \times 10^{3}$ has been used. If the Hubble parameter at the inflation period were of the order of the Planck scale, $H_{I} \sim M_{P}$, the present energy density (7.22) would be about the observed value $\left(M_{P} H_{0}\right)^{2}$. However, since the CMB fluctuation data give the constraint $H_{I}<3.6 \times 10^{-5} M_{p}$, (7.22) takes a much smaller value, $\rho_{0}<\frac{3}{32 \pi^{2}}\left(3.6 \times 10^{-5}\right)^{2}\left(M_{P} H_{0}\right)^{4} \sim\left(6.3 \times 10^{-3} \mathrm{meV}\right)^{4}$, than the desired one for the dark energy density. Moreover, unless we include possible effects of interactions, (7.23) cannot explain the negative pressure of the dark

\footnotetext{
${ }^{14}$ These expressions agree with the ones obtained in [14.
} 
energy. In the next section, we try to solve the first issue, namely, a possibility to enhance the present energy density to the desired value for the dark energy. The second issue of emergence of negative pressure due to interactions is left for future investigations.

\section{Models for Planckian era before inflation}

Let us now pursue a possibility that the energy density of a quantum field is enhanced due to the fluctuations created before inflation.

If the inflation period has a finite duration, i.e., a finite e-folding, the longwavelength modes, which are out of causal horizon throughout the inflation period, are not necessarily specified by the Bunch-Davies vacuum. If the universe was created quantum gravitationally, it is natural that quantum fields had fluctuations of the order of the Planck scale, and that the long-wavelength modes survive the inflation period. For instance, in the context of eternal inflation, our universe is generally surrounded by the region with the larger Hubble parameter, where larger fluctuations are generated.

As a simple example, we consider a model of a cosmic history with two inflation periods, i.e., the ordinary inflation with the Hubble parameter $H_{I}$ and another one with $H_{P} \sim M_{P}$ before the ordinary inflation 15 Because of the large Hubble parameter, the IR modes of the wave functions are enhanced to an order of $H_{P} \sim M_{P}$, and give the present energy density with an almost desired value, $\left(M_{P} H_{0}\right)^{2}$. In order to connect the scale factor $a$ and its derivative $a^{\prime}$ continuously between the two inflationary periods, we need to insert another period between them. A simple model is to insert the RD stage. The analysis of the energy and pressure densities of this model is performed in Appendix A. In the following, we study another model in which the curvature-dominated period is sandwiched between the two inflation periods.

\subsection{Double inflation model}

We consider a model with two inflation periods, connected by an intermediate curvature-dominated (CD) stage. We call the first inflation a pre-inflation. The scale factor is given by

$$
a(\eta)=\left\{\begin{array}{lll}
a_{\mathrm{PI}}(\eta)=-\frac{1}{H_{P} \eta} & \left(-\infty<\eta<\tilde{\eta}_{1}<0\right) & \text { (Pre-Inflation) } \\
a_{\mathrm{CD}}(\eta)=e^{\gamma \eta} & \left(0<\tilde{\eta}_{2}<\eta<\tilde{\eta}_{3}\right) & (\mathrm{CD}) \\
a_{\mathrm{Inf}}(\eta)=-\frac{1}{H_{I} \eta} & \left(\tilde{\eta}_{4}<\eta<\eta_{1}<0\right) & \text { (Inflation) }
\end{array}\right.
$$

\footnotetext{
${ }^{15} \mathrm{CMB}$ spectra in particular models that lead to two stages of inflation have been studied in 38,39 .
} 
The above periods are followed by the big bang universe (3.1) with the usual RD and MD periods. Here, $H_{P}$ is the Hubble parameter in the pre-inflation period. In the intermediate stage, one has $a_{\mathrm{CD}}(t)=\gamma t$. In order to satisfy the Friedman equations, (3.2) and (3.3), we need the energy density $\rho=3\left(\gamma M_{P}\right)^{2} a^{-2}$ and the pressure $p=-\rho / 3$, which has the same equation of state as the curvature. This model is studied as an example to connect the two inflationary periods continuously, and we do not discuss here how they are realized.

The matching conditions for the scale factor $a(\eta)$, i.e., continuity of $a$ and $a^{\prime}$, between the pre-inflation and CD periods are given by

$$
\begin{aligned}
& a_{\mathrm{PI}}\left(\tilde{\eta}_{1}\right)=a_{\mathrm{CD}}\left(\tilde{\eta}_{2}\right):-\frac{1}{H_{P} \tilde{\eta}_{1}}=e^{\gamma \tilde{\eta}_{2}} \\
& a_{\mathrm{PI}}^{\prime}\left(\tilde{\eta}_{1}\right)=a_{\mathrm{CD}}^{\prime}\left(\tilde{\eta}_{2}\right): \frac{1}{H_{P} \tilde{\eta}_{1}^{2}}=\gamma e^{\gamma \tilde{\eta}_{2}}
\end{aligned}
$$

Similarly, the conditions between the $\mathrm{CD}$ and inflation periods are

$$
\begin{aligned}
& a_{\mathrm{CD}}\left(\tilde{\eta}_{3}\right)=a_{\mathrm{Inf}}\left(\tilde{\eta}_{4}\right) \quad: \quad e^{\gamma \tilde{\eta}_{3}}=-\frac{1}{H_{I} \tilde{\eta}_{4}}, \\
& a_{\mathrm{CD}}^{\prime}\left(\tilde{\eta}_{3}\right)=a_{\mathrm{Inf}}^{\prime}\left(\tilde{\eta}_{4}\right) \quad: \quad \gamma e^{\gamma \tilde{\eta}_{3}}=\frac{1}{H_{I} \tilde{\eta}_{4}^{2}} .
\end{aligned}
$$

The conditions are solved as

$$
\begin{gathered}
\gamma=-\frac{1}{\tilde{\eta}_{1}}=-\frac{1}{\tilde{\eta}_{4}}, \\
e^{\gamma\left(\tilde{\eta}_{3}-\tilde{\eta}_{2}\right)}=\frac{H_{P}}{H_{I}} .
\end{gathered}
$$

The model has two additional parameters, $H_{P}$ and $\gamma$. As mentioned above, $H_{P}$ is assumed to be close to $M_{P} \cdot \gamma=-\tilde{\eta}_{4}^{-1}$ might be constrained by the condition that the inflation period has a sufficiently long duration to solve the horizon and flatness problems. The e-folding number of the inflation period is given by

$$
N_{e}=\ln \left(\frac{a_{\operatorname{Inf}}\left(\eta_{1}\right)}{a_{\operatorname{Inf}}\left(\tilde{\eta}_{4}\right)}\right)=\ln \left(\frac{\tilde{\eta}_{4}}{\eta_{1}}\right) .
$$

If we require that the fluctuations within the current horizon radius must be causally connected when the inflation begins, we have the condition $\tilde{\eta}_{4} / \eta_{1} \geq$ $\eta_{0} / \eta_{2}$. From (3.19), it gives $\left|\tilde{\eta}_{4}\right| \geq 2.1 \times 10^{27}\left|\eta_{1}\right|$, if the Hubble parameter is $H_{I}=3.6 \times 10^{-5} M_{P}$. In fact, $\left|\tilde{\eta}_{4}\right|$ can be made smaller and the beginning of the inflation can be made later if we solve the horizon and flatness problems together with the pre-inflation. But then the large fluctuations generated in the pre-inflation era enter the cosmic horizon earlier and may become observable in the CMB spectrum. We will discuss this issue again at the end of this section. 


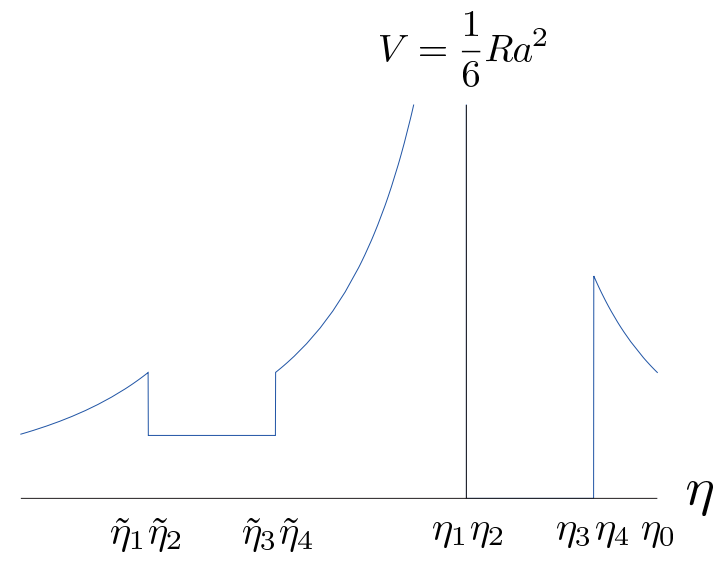

Figure 8: Potential of the wave equation in the double inflation model with an intermediate CD stage. It has a plateau with the height $\gamma^{2}$ in the CD period. The potential heights at the end of the pre-inflation and at the beginning of the inflation are the same and given by $2 / \tilde{\eta}_{1}^{2}=2 / \tilde{\eta}_{4}^{2}=2 \gamma^{2} . \eta_{0}$ is the present time.

The potential term of the wave equation (4.1) becomes

$$
\frac{1}{6} R a^{2}=\frac{a^{\prime \prime}}{a}=\left\{\begin{array}{lll}
2 / \eta^{2} & \left(-\infty<\eta<-\left|\tilde{\eta}_{1}\right|\right) & \text { (Pre-Inflation) } \\
\gamma^{2} & \left(\tilde{\eta}_{2}<\eta<\tilde{\eta}_{3}\right) & \text { (CD) }
\end{array}\right.
$$

followed by the potential (4.2) in the inflation, $\mathrm{RD}$, and $\mathrm{MD}$ periods. It is depicted in Figure 8. In the CD period, the potential has a plateau with the height $\gamma^{2}$. The solutions of the wave equations are given by

$$
\begin{aligned}
\chi_{\mathrm{PI}} & =\chi_{\mathrm{BD}}, \\
\chi_{\mathrm{CD}} & =\tilde{A} \chi_{\mathrm{pl}}+\tilde{B} \tilde{\chi}_{\mathrm{pl}}, \\
\chi_{\mathrm{Inf}} & =\tilde{C} \chi_{\mathrm{BD}}+\tilde{D} \chi_{\mathrm{BD}}^{*},
\end{aligned}
$$

in the pre-inflation, $\mathrm{CD}$, and inflation periods, respectively, and (4.5) and (4.6) in the $\mathrm{RD}$ and $\mathrm{MD}$ periods. Here, $\chi_{\mathrm{pl}}$ and $\tilde{\chi}_{\mathrm{pl}}$ are

$$
\chi_{\mathrm{pl}}=\left\{\begin{array}{ll}
\frac{1}{\sqrt{2 k}} e^{-\kappa \eta} & (k<\gamma) \\
\frac{1}{\sqrt{2 k}} e^{-i \omega \eta} & (k>\gamma)
\end{array} \quad, \quad \tilde{\chi}_{\mathrm{pl}}=\left\{\begin{array}{cc}
\frac{1}{\sqrt{2 k}} e^{\kappa \eta} & (k<\gamma) \\
\frac{1}{\sqrt{2 k}} e^{i \omega \eta} & (k>\gamma)
\end{array}\right.\right.
$$

with

$$
\begin{aligned}
\kappa & =\sqrt{\gamma^{2}-k^{2}}, \\
\omega & =\sqrt{k^{2}-\gamma^{2}} .
\end{aligned}
$$

The coefficients $\tilde{A}, \tilde{B}, \tilde{C}$, and $\tilde{D}$ are determined by the continuity of $\chi$ and 
$\chi^{\prime}$ as

$$
\begin{aligned}
& \left(\begin{array}{l}
\tilde{A} \\
\tilde{B}
\end{array}\right)=\frac{1}{2}\left(\begin{array}{l}
{\left[\left(1+i \frac{k}{\kappa}\right)\left(1-\frac{i}{k \tilde{\eta}_{1}}\right)-i \frac{k}{\kappa} \frac{1}{k^{2} \tilde{\eta}_{1}^{2}}\right] e^{\kappa \tilde{\eta}_{2}}} \\
{\left[\left(1-i \frac{k}{\kappa}\right)\left(1-\frac{i}{k \tilde{\eta}_{1}}\right)+i \frac{k}{\kappa} \frac{1}{k^{2} \tilde{\eta}_{1}^{2}}\right] e^{-\kappa \tilde{\eta}_{2}}}
\end{array}\right) e^{-i k \tilde{\eta}_{1}}, \\
& \left(\begin{array}{l}
\tilde{C} \\
\tilde{D}
\end{array}\right)=\frac{\kappa}{2 i k}\left(\begin{array}{c}
{\left[\left(1+i \frac{k}{\kappa}\right)\left(1+\frac{i}{k \tilde{\eta}_{4}}\right)-i \frac{k}{\kappa} \frac{1}{k^{2} \tilde{\eta}_{4}^{2}}\right] e^{-\kappa \tilde{\eta}_{3}+i k \tilde{\eta}_{4}}} \\
-\left[\left(1-i \frac{k}{\kappa}\right)\left(1-\frac{i}{k \tilde{\eta}_{4}}\right)+i \frac{k}{\kappa} \frac{1}{k^{2} \tilde{\eta}_{4}^{2}}\right] e^{-\kappa \tilde{\eta}_{3}-i k \tilde{\eta}_{4}}
\end{array}\right. \\
& \left.\begin{array}{r}
-\left[\left(1-i \frac{k}{\kappa}\right)\left(1+\frac{i}{k \tilde{\eta}_{4}}\right)+i \frac{k}{\kappa} \frac{1}{k^{2} \tilde{\eta}_{4}^{2}}\right] e^{\kappa \tilde{\eta}_{3}+i k \tilde{\eta}_{4}} \\
{\left[\left(1+i \frac{k}{\kappa}\right)\left(1-\frac{i}{k \tilde{\eta}_{4}}\right)-i \frac{k}{\kappa} \frac{1}{k^{2} \tilde{\eta}_{4}^{2}}\right] e^{\kappa \tilde{\eta}_{3}-i k \tilde{\eta}_{4}}}
\end{array}\right)\left(\begin{array}{c}
\tilde{A} \\
\tilde{B}
\end{array}\right),
\end{aligned}
$$

for $k<\gamma$. The results for $k>\gamma$ are obtained by replacing $\kappa$ by $i \omega$. The constants $A$ and $B$ in (4.5) are determined as

$$
\left(\begin{array}{l}
A \\
B
\end{array}\right)=\left(\begin{array}{cc}
\left(1-\frac{i}{k \eta_{1}}-\frac{1}{2 k^{2} \eta_{1}^{2}}\right) e^{-i k\left(\eta_{1}-\eta_{2}\right)} & \frac{1}{2 k^{2} \eta_{1}^{2}} e^{i k\left(\eta_{1}+\eta_{2}\right)} \\
\frac{1}{2 k^{2} \eta_{1}^{2}} e^{-i k\left(\eta_{1}+\eta_{2}\right)} & \left(1+\frac{i}{k \eta_{1}}-\frac{1}{2 k^{2} \eta_{1}^{2}}\right) e^{i k\left(\eta_{1}-\eta_{2}\right)}
\end{array}\right)\left(\begin{array}{l}
\tilde{C} \\
\tilde{D}
\end{array}\right)
$$

instead of (4.9). The coefficients $C$ and $D$ in (4.6) are determined as (4.10).

We first examine the IR behaviors of the wave function, and energy and pressure densities. As shown in Appendix B.2, the wave function in the IR region behaves as

$$
u^{\mathrm{IR}}=\frac{i}{\sqrt{2}} H_{P} k^{-3 / 2}+\mathcal{O}\left(k^{1 / 2}\right)
$$

in all the periods. As expected, (8.19) is amplified by the Hubble parameter $H_{P}$ in the pre-inflation period. Note that (8.19) is valid in the IR region $k<\gamma$ and $k<\eta^{-1}$. Substituting the IR wave function (8.19) into the expressions (5.1) and (5.3), the energy and pressure densities become

$$
\begin{aligned}
\rho^{\mathrm{IR}} & =\frac{H_{P}^{2}}{8 \pi^{2} a^{2}} \int_{0} d k\left[k+\mathcal{O}\left(k^{3}\right)\right], \\
p^{\mathrm{IR}} & =-\frac{1}{3} \frac{H_{P}^{2}}{8 \pi^{2} a^{2}} \int_{0} d k\left[k+\mathcal{O}\left(k^{3}\right)\right] .
\end{aligned}
$$

Compared to (5.5) and (5.6), they are enhanced by the factor $\left(H_{P} / H_{I}\right)^{2}$.

We next examine the UV behavior of the wave function. For $k>\gamma, k$ is above the plateau of the potential in the CD period. Hence, wave functions with $k>\gamma$ are not affected much by this potential, and reduce to the previous one without the pre-inflation and the CD periods. Indeed, eqs. (8.16) and (8.17), with $\kappa$ replaced by $i \omega$, show that the coefficients approach $\tilde{C}=1$ and $\tilde{D}=0$ for large $k$. If we smoothly connect the potential in different periods, $\tilde{D}$ approaches 
0 much faster. Consequently, the IR amplification of the wave function in the pre-inflation period terminates at $k \sim \gamma$.

Figure 9 shows the integrands $\rho(k)$ and $p(k)$. The parameters are taken to be the same as those in the third row of Figure 2. The upper figures show that $\rho(k)$ and $p(k)$ acquire a new peak in the IR region $0<k<\gamma$, which is generated by the pre-inflation. It is also confirmed in the lower figures. The upper figures also indicate that the integrands reduce to the previous ones for larger $k$.

It is important to note that the new peak is located in the region $k \in[0, \gamma]$, and independent of $\eta$. The behavior is different from the peaks generated in the ordinary inflation, which are dependent on $\eta$. The difference comes from the difference of scales $\left|\tilde{\eta}_{1}\right| \gg\left|\eta_{1}\right|$. Since the potential peak of the ordinary inflation $2 /\left|\eta_{1}\right|^{2}$ is high, the modes up to large $k=\left|\eta_{1}\right|^{-1}$ are amplified. As we discussed before, the integrands $\rho(k)$ and $p(k)$ decrease and oscillate with the period $\Delta k=\pi / \eta$, and this $\eta$-dependent behavior shows up in the region $k \in\left[0,\left|\eta_{1}\right|^{-1}\right]$ since $\pi / \eta<\left|\eta_{1}\right|^{-1}$. On the contrary, only the modes with $k<\gamma=\left|\tilde{\eta}_{1}\right|^{-1}$ are affected by the pre-inflation. Then the $\eta$-dependent behavior does not show up in the region $k \in\left[0,\left|\tilde{\eta}_{1}\right|^{-1}\right]$ if $\pi / \eta>\left|\tilde{\eta}_{1}\right|^{-1}$. In other words, the modes enhanced in the inflationary period are entering the horizon after the big bang, but the modes enhanced in the pre-inflation period are still out of the horizon. It causes the difference of the behaviors of the integrands $\rho(k)$ and $p(k)$, and consequently the big difference of the time evolution of the energy densities as explained in Section 8.2 .

We now perform the integration of $\rho(k)$ and $p(k)$ over $k$. When $\eta<\left|\tilde{\eta}_{1}\right|$, the integrations are dominated by the large IR peak generated by the pre-inflation period and given by

$$
\begin{aligned}
\rho^{\text {IRpeak }} & \sim \frac{H_{P}^{2}}{8 \pi^{2} a^{2}} 2 \int_{0}^{\gamma / 2} d k k=\frac{H_{P}^{2}}{32 \pi^{2} a^{2}} \gamma^{2}, \\
p^{\text {IRpeak }} & =-\frac{1}{3} \rho^{\text {IRpeak }},
\end{aligned}
$$

where we approximated the position of the peak at $\gamma / 2$. The factor 2 comes from an approximation that the peak has a form of an isosceles triangle. They give the equation of state $w=p / \rho=-1 / 3$. This is consistent with the fact that the time dependence of (8.22) and (8.23) is $a^{-2}$. Neither the integrand nor the upper bound $\gamma / 2$ depends on $\eta$. It is due to the condition of $\eta<\left|\tilde{\eta}_{1}\right|$. Namely, the amplified waves in the pre-inflation period are still out of the horizon and are not yet affected by the oscillatory behaviors of quantum waves.

When time passes and $\eta$ becomes larger than $\gamma^{-1}=\left|\tilde{\eta}_{1}\right|$, the oscillating and decreasing behavior of the integrand with the period $\Delta k=\pi / \eta$ enters the 

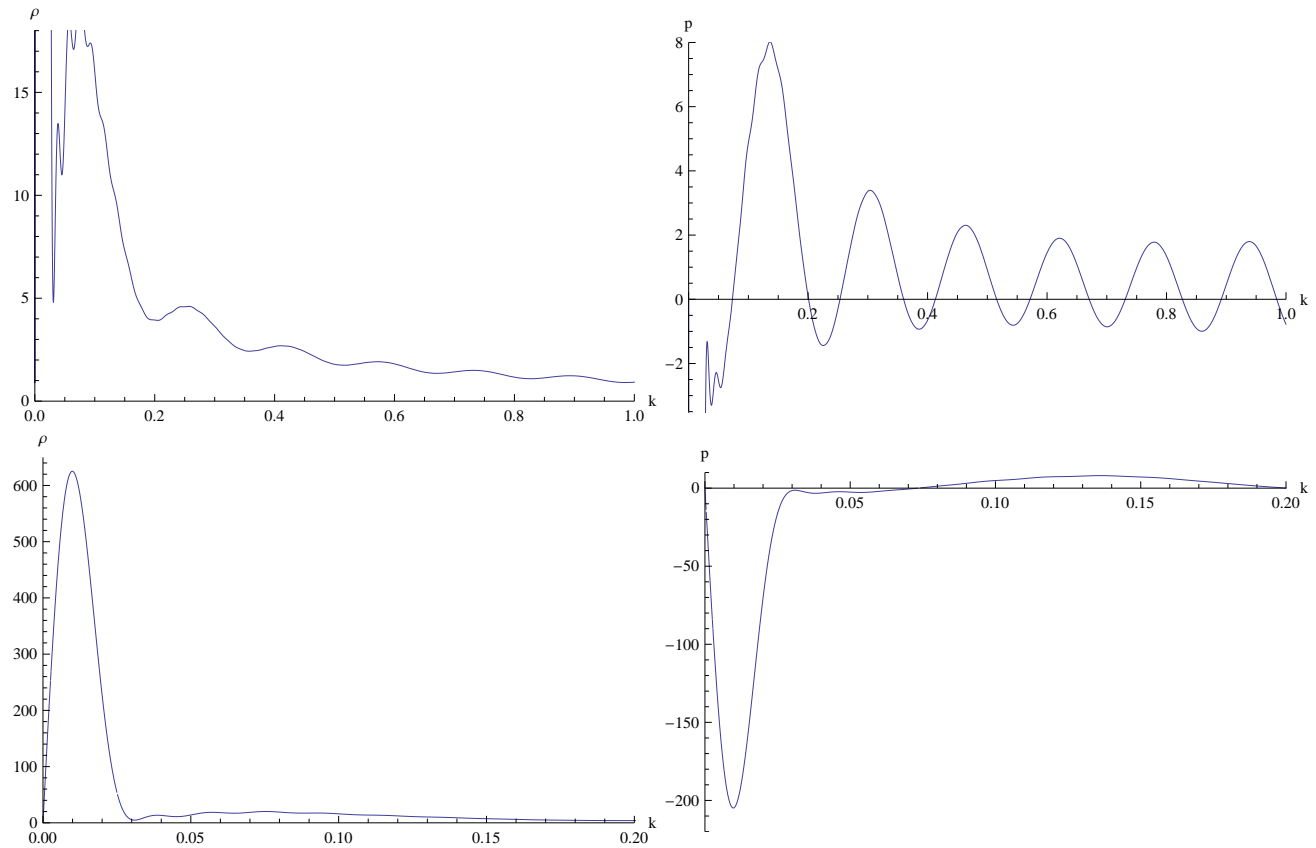

Figure 9: The integrands of $\rho(k)$ and $p(k)$ in the RD period, caused by the double inflation model. As in the third row of Figure 2, we take $\eta_{2}=-\eta_{1}=1$ and $\eta=20$. The other parameters are set to be $\gamma^{-1}=-\tilde{\eta}_{1}=-\tilde{\eta}_{4}=40$ and $H_{P} / H_{I}=16$. Accordingly, $\tilde{\eta}_{3}-\tilde{\eta}_{2}=\gamma^{-1} \ln \left(H_{P} / H_{I}\right)=40 \ln (16) \sim 111$. Note that these values of $\gamma$ and $H_{P}$ are not realistic, but just chosen here to draw the figures. In the upper figures, the scale of the axes is taken to be the same as in the third row of Figure 2, The lower figures magnify the IR region of the upper figures. We can see modifications of $\rho$ and $p$ in the IR region by the pre-inflation. 
interval $0<k<\gamma$ of the IR peak, and $\eta$ dependence other than $a^{-2}$ arises. The results approach those in Section 7.2 , with $H_{I}$ replaced by $H_{P}$, if the background geometry of the MD period continues.

The energy density (8.22) depends on the two parameters of the model, $H_{P}$ and $\gamma=\left|\tilde{\eta}_{4}\right|^{-1}$. We consider natural values for these parameters. Since we consider the Planckian era, $H_{P}$ is close to $M_{P}$. The other parameter, $\gamma=\left|\tilde{\eta}_{4}\right|^{-1}$, is related to the e-folding number (8.8). The necessary e-folding number to solve the horizon and flatness problems can be gained together with the pre-inflation era. However, if we chose $\gamma>2 \pi \eta_{0}^{-1}$, the enormously amplified wave functions by the pre-inflation, which lie in the region $k \in[0, \gamma]$, would enter the current horizon, which corresponds to the comoving wave number $k=2 \pi \eta_{0}^{-1}$. Such modes within the horizon could be detected. For instance, they may generate additional CMB fluctuations and receive strong constraints from the observed data. We then choose $\gamma \leq 2 \pi \eta_{0}^{-1}$

To conclude this section, if we set the parameters of the model as $H_{P}=$ $M_{P}$ and $\gamma=2 \pi \eta_{0}^{-1}$, (8.22) gives $\rho_{0}=\left(M_{P} H_{0}\right)^{2} / 32$ at present, where $H_{0}=$ $2\left(a_{0} \eta_{0}\right)^{-1}$ is used. It becomes close to the desired order of magnitude of the dark energy $\sim\left(M_{P} H_{0}\right)^{2}$ at present.

\subsection{Time evolution of energy and pressure densities}

We then study how the energy and pressure densities, caused by the double inflation model with $H_{P}=M_{P}$ and $\gamma=2 \pi \eta_{0}^{-1}$, evolve with time.

The energy density generated by the pre-inflation is estimated in (8.22). Substituting $H_{P}=M_{P}$ and $\gamma=2 \pi \eta_{0}^{-1}$, it becomes

$$
\rho_{\text {pre-inf }} \simeq \frac{1}{8} M_{P}^{2} \frac{1}{a^{2} \eta_{0}^{2}}=\left\{\begin{array}{ll}
\frac{1}{32}\left(M_{P} H\right)^{2}\left(\frac{a_{\mathrm{eq}}}{a_{0}}\right)\left(\frac{a_{\mathrm{BB}}}{a_{\mathrm{eq}}}\right)^{2}\left(\frac{a_{\mathrm{BB}}}{a}\right)^{2} & \text { (Inflation) } \\
\frac{1}{32}\left(M_{P} H\right)^{2}\left(\frac{a_{\mathrm{eq}}}{a_{0}}\right)\left(\frac{a}{a_{\mathrm{eq}}}\right)^{2} & (\mathrm{RD}) \\
\frac{1}{32}\left(M_{P} H\right)^{2}\left(\frac{a}{a_{0}}\right) & (\mathrm{MD})
\end{array} .\right.
$$

In the last equality, we used (3.12) and factored out $H^{2}$, which is proportional to $a^{-3}, a^{-4}$, and $a^{0}$ at the $\mathrm{MD}, \mathrm{RD}$, and inflation periods, respectively. Here, $\mathrm{BB}$ stands for the big bang, the time when the RD period begins. eq and 0 stand for the matter-radiation equality and the present. The equation of state is given by $w=-1 / 3$.

On the other hand, the energy density produced by the standard inflation is given by (7.8) in the RD period, and the sum of (7.18) and (7.20) in the MD 


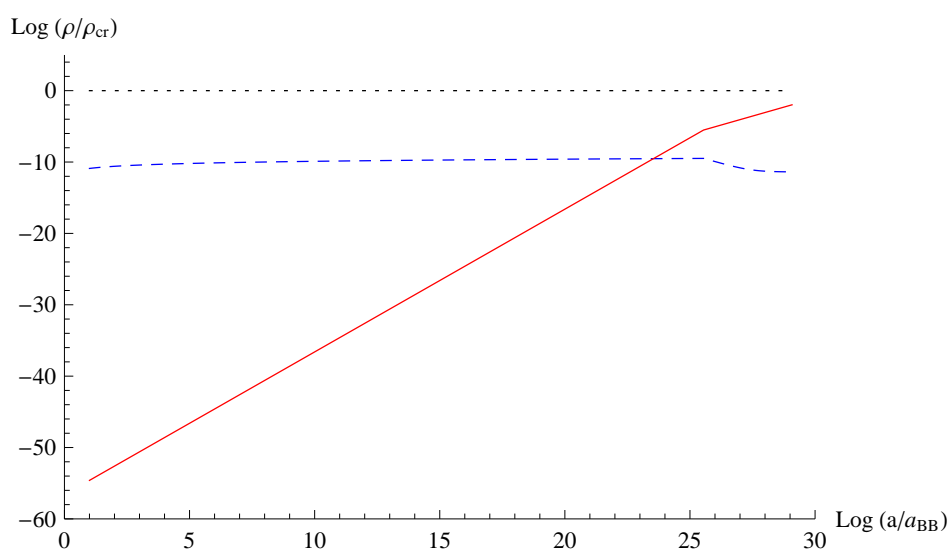

Figure 10: Time evolution of the energy density of a quantum field in the $\mathrm{RD}$ and MD periods. The energy density divided by the critical energy is depicted against the scale factor $a / a_{\mathrm{BB}}$ in the logarithmic scale. The solid and dashed lines represent the ratios of the energy density generated in the pre-inflation and inflation, respectively, to the critical energy density. The dotted line corresponds to the critical density. The parameters are taken to be $H_{I} / M_{P}=3.6 \times 10^{-5}, a_{\mathrm{eq}} / a_{\mathrm{BB}}=3.5 \times 10^{25}$, and $a_{0} / a_{\mathrm{BB}}=1.2 \times 10^{29}$.

period. It is written as

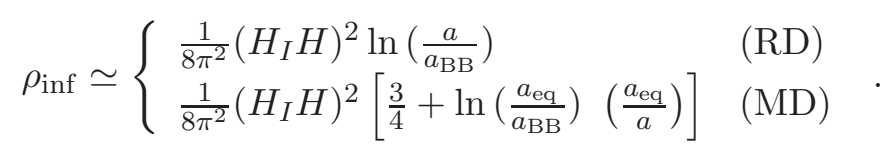

The equation of state is $w=1 / 3$ in the $\mathrm{RD}$ period and approaches $w=0$ in the MD period.

Figure 10 shows the time evolution of the ratios of the energy densities, (8.24) and (8.25), to the critical energy density

$$
\rho_{\mathrm{cr}}=3\left(M_{P} H\right)^{2}
$$

in the RD and MD periods. The ratios are depicted as a function of $a / a_{\mathrm{BB}}$ in the logarithmic scale.

The ratio $\rho_{\text {inf }} / \rho_{\text {cr }}$ is almost constant since both $\rho_{\text {inf }}$ and $\rho_{\text {cr }}$ have a time dependence of $H^{2}$. $\rho_{\text {inf }}$ is always smaller than the critical value $\rho_{\text {cr }}$ by the factor of $\left(H_{I} / M_{P}\right)^{2}$. On the contrary, $\rho_{\text {pre-inf }}$ has the time dependence shown in (8.24). The ratio $\rho_{\text {pre-inf }} / \rho_{\text {cr }}$ decreases in the inflationary period and takes a small value when the $\mathrm{RD}$ period starts. But it grows after that. $\rho_{\text {pre-inf }}$ becomes larger than $\rho_{\text {inf }}$ at $a_{*}=3.5 \times 10^{23} a_{\mathrm{BB}}=2.9 \times 10^{-6} a_{0}$, and eventually approaches $\rho_{\text {cr }}$. Hence, the energy density of a quantum field is dominated by 
the contribution $\rho_{\text {inf }}$ before $a=a_{*}$ and by $\rho_{\text {pre-inf }}$ after that. The equation of state for the sum of these two contributions changes from $w_{\mathrm{vac}}=1 / 3$ to $w_{\mathrm{vac}}=-1 / 3$ around $a=a_{*}$.

In the future, as we mentioned below (8.22), the wave functions amplified in the pre-inflation enter the horizon and the energy density evolves as $\left(M_{P} H\right)^{2} / 32$. If the background geometry of the MD period continues, the equation of state eventually becomes $w_{\mathrm{vac}}=0$. But if the induced energy dominates the critical density, we need to take back reactions into account, and the equation of state will also be changed accordingly.

If we take $\left(H_{P}, \gamma\right)=\left(M_{P}, 2 \pi \eta_{0}^{-1}\right)$, the energy density of a quantum field becomes $\left(M_{P} H_{0}\right)^{2} / 32$ at present. It is still 96 times smaller than the critical value (8.26). In order to obtain a larger value, we may make either $H_{P}$ larger or $\gamma=\left|\tilde{\eta}_{4}\right|^{-1}$ larger. For simplicity, we fix $H_{I}=3.6 \times 10^{-5} M_{P}$, and, accordingly, the conformal time at the end of the standard inflation $\eta_{1}$ is fixed. If we write $\left|\tilde{\eta}_{4}\right|^{-1}=2 \pi \eta_{0}^{-1} x, x$ is related to the e-folding number during the ordinary inflation as $x=\frac{1}{2 \pi} \frac{\eta_{0}}{\eta_{2}} \frac{\eta_{1}}{\tilde{\eta}_{4}}=e^{61.1-N_{e}} \cdot x=\frac{1}{2 \pi}$ corresponds to the lower value of the e-folding in the ordinary scenario of the inflation. Then the energy density (8.22) at present becomes

$$
\rho_{0}=\frac{e^{2\left(61.1-N_{e}\right)}}{32}\left(H_{P} H_{0}\right)^{2} .
$$

In order to make it comparable to the critical density, we need $x H_{P} / M_{P} \sim$ 10. The condition can be satisfied by setting, e.g., $\left(H_{P}, N_{e}\right)=\left(M_{P}, 58.8\right)$, $\left(5 M_{P}, 60.4\right)$, or $\left(10 M_{P}, 61.1\right)$. These numbers should not be taken at face value since they are sensitive to details of the cosmic history such as the intermediate stage between the two inflations, or interactions neglected in the analysis of this paper.

\section{Conclusions and discussion}

In this paper, we calculated the time evolution of the energy-momentum tensor of a minimally-coupled massless scalar field throughout the history of the universe. We considered two types of cosmic histories. The first one is the standard cosmology model, starting from the inflation and followed by the RD and MD periods. To perform quantization, the Bunch-Davies initial condition is imposed on the field in the inflation period. Due to the fact that inflation produces fluctuations of the order $H_{I}$, the energy density of a quantum field becomes of the order $\rho_{\text {inf }} \sim\left(H_{I} H\right)^{2}$ where $H$ and $H_{I}$ are the Hubble parameters at each moment and in the inflation period. The evolution of $\rho_{\text {inf }}$ is given by eq. (8.25) and its ratio to the critical density is depicted as the dashed line 
in Figure 10. The ratio is almost constant but the magnitude is much smaller than the critical density. The equation of state evolves from a negative value to $w=1 / 3$ in the RD period, and from $1 / 3$ to $w=0$ in the MD period.

The second type of model we considered is a double inflation model, in which we assume another inflation, which we call a pre-inflation, exists before the ordinary inflation starts. The present energy density of the fluctuations generated in the pre-inflation is enhanced to an order of $\rho_{\text {pre-inf }} \sim\left(H_{P} H_{0}\right)^{2}$ and is comparable to the critical density if $H_{P} \sim M_{P}$. Here, $H_{P}$ is the Hubble parameter of the pre-inflation and $M_{P}$ is the Planck scale. The evolution of $\rho_{\text {pre-inf }}$, given by eq. (8.24), is different from $\rho_{\text {inf }}$ because the amplified wave functions in the pre-inflation have larger wavelengths and have not entered the horizon yet. The ratio to the critical density is depicted by the solid line in Figure 10. The equation of state is given by $w=p / \rho=-1 / 3$. It is negative, but cannot drive the acceleration of our universe within the free field approximation.

Our result suggests that fluctuations generated before the ordinary inflation could have an important effect on the present universe. We have considered the double inflation models as a simple explicit example in this paper. There are theoretically well-motivated models in the context of eternal inflation. In a theory with a metastable vacuum, universes are created by bubble nucleation [17]; such universes are surrounded by de Sitter space (the ancestor vacuum) with the Hubble parameter being supposedly much higher than the one for the ordinary inflation $H_{I}$. The vacuum state in such a universe can be defined by the Euclidean prescription, and is different from the Bunch-Davies vacuum for ordinary inflation [40. It is an important problem to find the renormalized energy-momentum tensors in such a universe, extending the analysis of this paper.

In this work, we have not considered back reactions from the quantum fluctuations to the geometry. In recent papers [41, 42], in which the effect of a large number of fields (Kaluza-Klein and string states) on the CMB fluctuations was studied, the authors made an interesting proposal that the vacuum energy from these fluctuations itself drives the acceleration in inflation. Back reactions to the geometry in this context are being studied by these authors. In our work, the energy density of the fluctuations is much smaller than the critical value during most of the periods in the cosmic history. However, it becomes relevant at the very early universe when the Hubble parameter is close to the Planck scale, and also at times later than the present where the vacuum fluctuation found in this paper starts to be dominant. It would be a very interesting theoretical problem to study the late time behavior to understand the fate of the universe. In this region, the interplay among the scale factor, the IR 
behavior of the wave function, and the Bunch-Davies initial condition should determine the dynamics of the universe self-consistently.

We solved the evolution equation in the free field approximation. The system is very simple, yet the evolution of the energy density of a quantum field summarized in Figure 10 is nontrivial. Then what will happen if we include interactions among them? Although there have been debates on the physical effect of quantum loops in the de Sitter background (see, e.g., 43. and references therein), it would be reasonable to assume that massive (including light but not exactly massless) fields will reach the interacting Hartle-Hawking vacuum [44]. If this is the case, the effect of loops modifies the numerical coefficients in front of $\rho$, but not their qualitative behaviors.

As a final remark, we mention a possible scenario of how interactions change the equation of state. As is well known in the Bogoliubov theory of superfluidity, quantum Bose gases fall into a macroscopic state in the presence of interactions. If the interaction is attractive, the system is unstable with negative pressure. Let us assume here that condensation occurs in our system. Indeed, the scalar field has gravitational interaction. Since the gravitational interaction is very weak and the energy density is also very low, it will take a long time for the condensation to occur. Once the condensation occurs, the interaction energy dominates the kinetic energy and the equation of state will be changed from the free case considered in the present paper to the interaction dominated form. Since the gravitational interaction is attractive, the negative pressure is expected to arise. It is interesting to understand the time scale of the condensation dynamics and evolutions of the energy-momentum tensor modified by gravitational interactions. We will investigate these issues in future publications.

\section{Acknowledgements}

The authors would like to thank Yoshinobu Habara, Hikaru Kawai, Hiroyuki Kitamoto, Yoshihisa Kitazawa, and Masao Ninomiya for valuable comments and discussions, and the participants in the KEK theory workshop held on February 18-21, 2014, for valuable discussions. This work is supported in part by Grant-in-Aid for Scientific Research (No. 23244057, 23540329, 24540279 and 24540293) from the Japan Society for the Promotion of Science. This work is also partially supported by "The Center for the Promotion of Integrated Sciences (CPIS)" of Sokendai. 


\section{A Double inflation model with an intermediate RD stage}

In this appendix, we consider a double inflation model, as in Section 8.1, but with the RD period as an intermediate stage. We call the fist inflation a preinflation period, and the subsequent intermediate RD stage a pre-RD period. The scale factor $a(\eta)$ is given by

$$
a(\eta)=\left\{\begin{array}{lll}
a_{\mathrm{PI}}(\eta)=-\frac{1}{H_{P} \eta} & \left(-\infty<\eta<\eta_{1}^{\prime}<0\right) & \text { (Pre-Inflation) } \\
a_{\mathrm{PR}}(\eta)=\alpha^{\prime} \eta & \left(0<\eta_{2}^{\prime}<\eta<\eta_{3}^{\prime}\right) & \text { (Pre-RD) } \\
a_{\mathrm{Inf}}(\eta)=-\frac{1}{H_{I} \eta} & \left(\eta_{4}^{\prime}<\eta<\eta_{1}<0\right) & \text { (Inflation) }
\end{array}\right.
$$

instead of (8.1). The matching conditions, i.e., the continuity of $a$ and $a^{\prime}$, between the pre-inflation and pre-RD periods are given by

$$
\begin{aligned}
& a_{\mathrm{PI}}\left(\eta_{1}^{\prime}\right)=a_{\mathrm{PR}}\left(\eta_{2}^{\prime}\right):-\frac{1}{H_{P} \eta_{1}^{\prime}}=\alpha^{\prime} \eta_{2}^{\prime}, \\
& a_{\mathrm{PI}}^{\prime}\left(\eta_{1}^{\prime}\right)=a_{\mathrm{PR}}^{\prime}\left(\eta_{2}^{\prime}\right): \frac{1}{H_{P} \eta_{1}^{\prime 2}}=\alpha^{\prime},
\end{aligned}
$$

and those between the pre-RD and inflation periods are

$$
\begin{aligned}
& a_{\mathrm{PR}}\left(\eta_{3}^{\prime}\right)=a_{\mathrm{Inf}}\left(\eta_{4}^{\prime}\right) \quad: \quad \alpha^{\prime} \eta_{3}^{\prime}=-\frac{1}{H_{I} \eta_{4}^{\prime}} \\
& a_{\mathrm{PR}}^{\prime}\left(\eta_{3}^{\prime}\right)=a_{\mathrm{Inf}}^{\prime}\left(\eta_{4}^{\prime}\right) \quad: \quad \alpha^{\prime}=\frac{1}{H_{I} \eta_{4}^{\prime 2}}
\end{aligned}
$$

They are solved as

$$
\begin{aligned}
-\eta_{1}^{\prime} & =\eta_{2}^{\prime} \\
\eta_{3}^{\prime} & =-\eta_{4}^{\prime} \\
\frac{\eta_{1}^{\prime}}{\eta_{4}^{\prime}} & =\sqrt{\frac{H_{I}}{H_{P}}} .
\end{aligned}
$$

Note that A.8) gives the constraint $\eta_{4}^{\prime} / \eta_{1}^{\prime}>1.7 \times 10^{2} \gg 1$.

The potential for the wave equation (4.1) becomes

$$
\frac{1}{6} R a^{2}=\frac{a^{\prime \prime}}{a}=\left\{\begin{array}{ll}
2 / \eta^{2} & (\text { Pre-Inflation }) \\
0 & (\text { Pre-RD })
\end{array},\right.
$$

followed by (4.2). It is depicted in Figure 11]. Since the potential vanishes in the RD period, the pre-RD period gives a chasm in the potential. The solution for the wave equation is given as

$$
\chi_{\mathrm{PR}}=A^{\prime} \chi_{\mathrm{PW}}+B^{\prime} \chi_{\mathrm{PW}}^{*}
$$




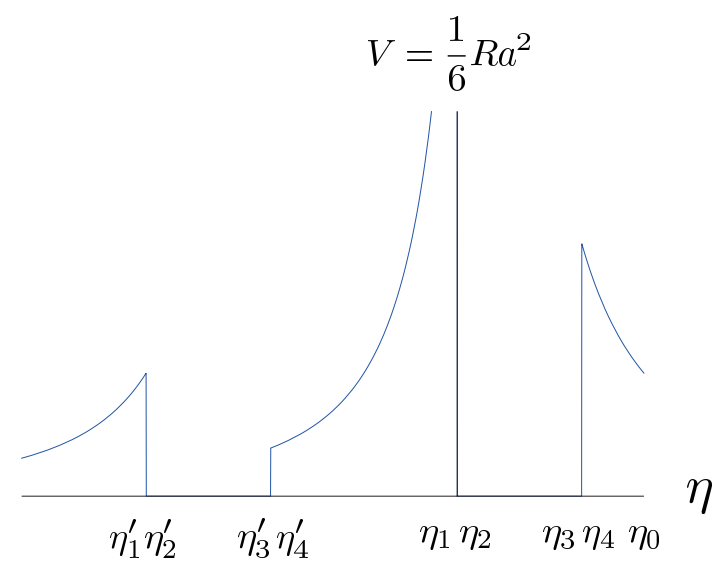

Figure 11: Potential for the wave equation in the double inflation model with the intermediate RD stage. $\eta_{1}^{\prime}$ to $\eta_{4}^{\prime}$ and $\eta_{1}$ to $\eta_{4}$ are the edges of the preinflation, pre-RD, inflation, $\mathrm{RD}$, and $\mathrm{MD}$ periods. $\eta_{0}$ is the present time.

in the pre-RD period. The wave functions in the pre-inflation and inflation periods are given by (8.10) and (8.12), with $\tilde{C}$ and $\tilde{D}$ replaced by $C^{\prime}$ and $D^{\prime}$, and those in the RD and MD periods are given by (4.5) and (4.6).

The constants $A^{\prime}, B^{\prime}, C^{\prime}$, and $D^{\prime}$ are determined by the matching conditions for the wave functions, i.e., the continuity of $\chi$ and $\chi^{\prime}$, as

$$
\begin{gathered}
\left(\begin{array}{c}
A^{\prime} \\
B^{\prime}
\end{array}\right)=\left(\begin{array}{c}
\left(1-\frac{i}{k \eta_{1}^{\prime}}-\frac{1}{2 k^{2} \eta_{1}^{\prime 2}}\right) e^{i k \eta_{2}^{\prime}} \\
\frac{1}{2 k^{2} \eta_{1}^{\prime 2}} e^{-i k \eta_{2}^{\prime}}
\end{array}\right) e^{-i k \eta_{1}^{\prime}}, \\
\left(\begin{array}{c}
C^{\prime} \\
D^{\prime}
\end{array}\right)=\left(\begin{array}{cc}
\left(1+\frac{i}{k \eta_{4}^{\prime}}-\frac{1}{2 k^{2} \eta_{4}^{\prime 2}}\right) e^{i k\left(\eta_{4}^{\prime}-\eta_{3}^{\prime}\right)} & -\frac{1}{2 k^{2} \eta_{4}^{\prime 2}} e^{i k\left(\eta_{4}^{\prime}+\eta_{3}^{\prime}\right)} \\
-\frac{1}{2 k^{2} \eta_{4}^{\prime 2}} e^{-i k\left(\eta_{4}^{\prime}+\eta_{3}^{\prime}\right)} & \left(1-\frac{i}{k \eta_{4}^{\prime}}-\frac{1}{2 k^{2} \eta_{4}^{\prime 2}}\right) e^{-i k\left(\eta_{4}^{\prime}-\eta_{3}^{\prime}\right)}
\end{array}\right)\left(\begin{array}{c}
A^{\prime} \\
B^{\prime}
\end{array}\right),
\end{gathered}
$$

which have exactly the same form as (4.9) and (4.10) with $A, B, C, D$, and $\eta_{1}, \ldots, \eta_{4}$ replaced by those with a prime. The coefficients $A, B, C$, and $D$ here are determined by (8.18), with $\tilde{C}$ and $\tilde{D}$ replaced by $C^{\prime}$ and $D^{\prime}$, and (4.10).

The IR behaviors of the wave function can be estimated as before. As will be shown in Appendix B.3, the IR behavior (8.19) is held in all the periods. The enhancement of the integrand for the energy and pressure densities, (8.20) and (8.21), is obtained accordingly.

The UV behavior, on the other hand, has a slightly complicated structure. Let us recall that (A.8) gives $\left|\eta_{1}^{\prime}\right|^{-1} \gg\left|\eta_{4}^{\prime}\right|^{-1}$. It follows that the potential height at the end of the pre-inflation and that at the beginning of the inflation in Figure 11 are largely different. For $k<\left|\eta_{4}^{\prime}\right|^{-1}$, the above estimations of the IR behaviors are valid, and the large enhancement (8.20) and (8.21) is obtained. 
For $k>\left|\eta_{1}^{\prime}\right|^{-1}$, since $k$ is above the potential throughout the pre-inflation and pre-RD periods, the results are not affected by those periods, and are reduced to the original ones without the pre-inflation. However, in the present model, there exist modes with $\left|\eta_{4}^{\prime}\right|^{-1}<k<\left|\eta_{1}^{\prime}\right|^{-1}$, which are modified from the original ones, but are not enhanced as largely as the IR modes.

We now examine the modes with $\left|\eta_{4}^{\prime}\right|^{-1}<k<\left|\eta_{1}^{\prime}\right|^{-1}$. For $\eta_{3}^{\prime-1}=\left|\eta_{4}^{\prime}\right|^{-1}<$ $k<\left|\eta_{1}^{\prime}\right|^{-1}=\eta_{2}^{\prime-1}$, by using the leading terms in (A.11), (A.12), and (8.18), one obtains

$$
\begin{aligned}
& \left(\begin{array}{l}
A \\
B
\end{array}\right) \sim \frac{-1}{2 k^{2} \eta_{1}^{2}}\left(\begin{array}{cc}
1 & -1 \\
-1 & 1
\end{array}\right)\left(\begin{array}{l}
e^{i k\left(\eta_{4}^{\prime}-\eta_{3}^{\prime}\right)} \\
\end{array} e^{-i k\left(\eta_{4}^{\prime}-\eta_{3}^{\prime}\right)}\right) \frac{-1}{2 k^{2} \eta_{1}^{\prime 2}}\left(\begin{array}{c}
1 \\
-1
\end{array}\right) \\
& =\frac{1}{2 k^{4} \eta_{1}^{2} \eta_{1}^{\prime 2}}\left(\begin{array}{c}
\cos k\left(\eta_{4}^{\prime}-\eta_{3}^{\prime}\right) \\
-\cos k\left(\eta_{4}^{\prime}-\eta_{3}^{\prime}\right)
\end{array}\right) \text {. }
\end{aligned}
$$

In the transitions from the pre-inflation to pre-RD period, and from the inflation to the RD period, the complete scattering takes place due to the high potential barrier, while in the transition from the pre-RD to inflation period, the scattering amplitude receives the phases that differ much from unity. Then the wave function (4.5) becomes

$$
\begin{aligned}
\chi_{\mathrm{RD}} & \sim \frac{1}{2 k^{4} \eta_{1}^{2} \eta_{1}^{\prime 2}} \cos \left(k\left(\eta_{4}^{\prime}-\eta_{3}^{\prime}\right)\right) \frac{-2 i}{\sqrt{2 k}} \sin (k \eta) \\
& =-\left(k \eta_{4}^{\prime}\right)^{-2} \cos \left(k\left(\eta_{4}^{\prime}-\eta_{3}^{\prime}\right)\right) \cdot \frac{1}{\sqrt{2 k}} \frac{i \eta_{4}^{\prime 2}}{k^{2} \eta_{1}^{2} \eta_{1}^{\prime 2}} \sin (k \eta)
\end{aligned}
$$

where the last factor represents the wave function for $k<\left|\eta_{4}^{\prime}\right|^{-1}$, which involves the enhancement factor $\left(\eta_{4}^{\prime} / \eta_{1}^{\prime}\right)^{2}$ caused by the pre-inflation, as shown in (B.38). Eq. A.16) involves an extra factor, $-\left(k \eta_{4}^{\prime}\right)^{-2} \cos \left(k\left(\eta_{4}^{\prime}-\eta_{3}^{\prime}\right)\right)$. Hence, the integrand for the energy and pressure densities in $\left|\eta_{4}^{\prime}\right|^{-1}<k<\left|\eta_{1}^{\prime}\right|^{-1}$ receive the extra factor $\left(k \eta_{4}^{\prime}\right)^{-4} \cos ^{2}\left(k\left(\eta_{4}^{\prime}-\eta_{3}^{\prime}\right)\right)$, compared to the one in the IR region $k<\left|\eta_{4}^{\prime}\right|^{-1}$. This extra factor decreases as $k^{-4}$, and oscillates with the period $\pi /\left|\eta_{4}^{\prime}-\eta_{3}^{\prime}\right|=\pi /\left|2 \eta_{4}^{\prime}\right|$.

Figure 12 shows the integrands $\rho(k)$ and $p(k)$. The parameters are taken to be the same as those in the third row of Figure 2, From the lower figures, one can see that the large IR peak lies in $0<k<\frac{\pi}{2}\left|\eta_{4}^{\prime}\right|^{-1}$, but the small modification of the integrand continues until $k \sim 5\left|\eta_{1}^{\prime}\right|^{-1}$. The upper figures show that, above $k \sim 5\left|\eta_{1}^{\prime}\right|^{-1}$, the integrands reduce to the ones in the third row of Figure 2, If the background geometry is smoothly connected, the UV tail of $\rho(k)$ and $p(k)$ will decrease more rapidly above $k=\left|\eta_{1}^{\prime}\right|^{-1}$. However, the energy and pressure densities in the region $k \in\left[\left|\eta_{4}^{\prime}\right|^{-1},\left|\eta_{1}^{\prime}\right|^{-1}\right]$ behave quite non-trivially. Due to the inequality $\left|\eta_{1}^{\prime}\right|^{-1} \gg\left|\eta_{4}^{\prime}\right|^{-1}$ from (A.8), this region is very large and the total energy and pressure receive substantial modifications. 


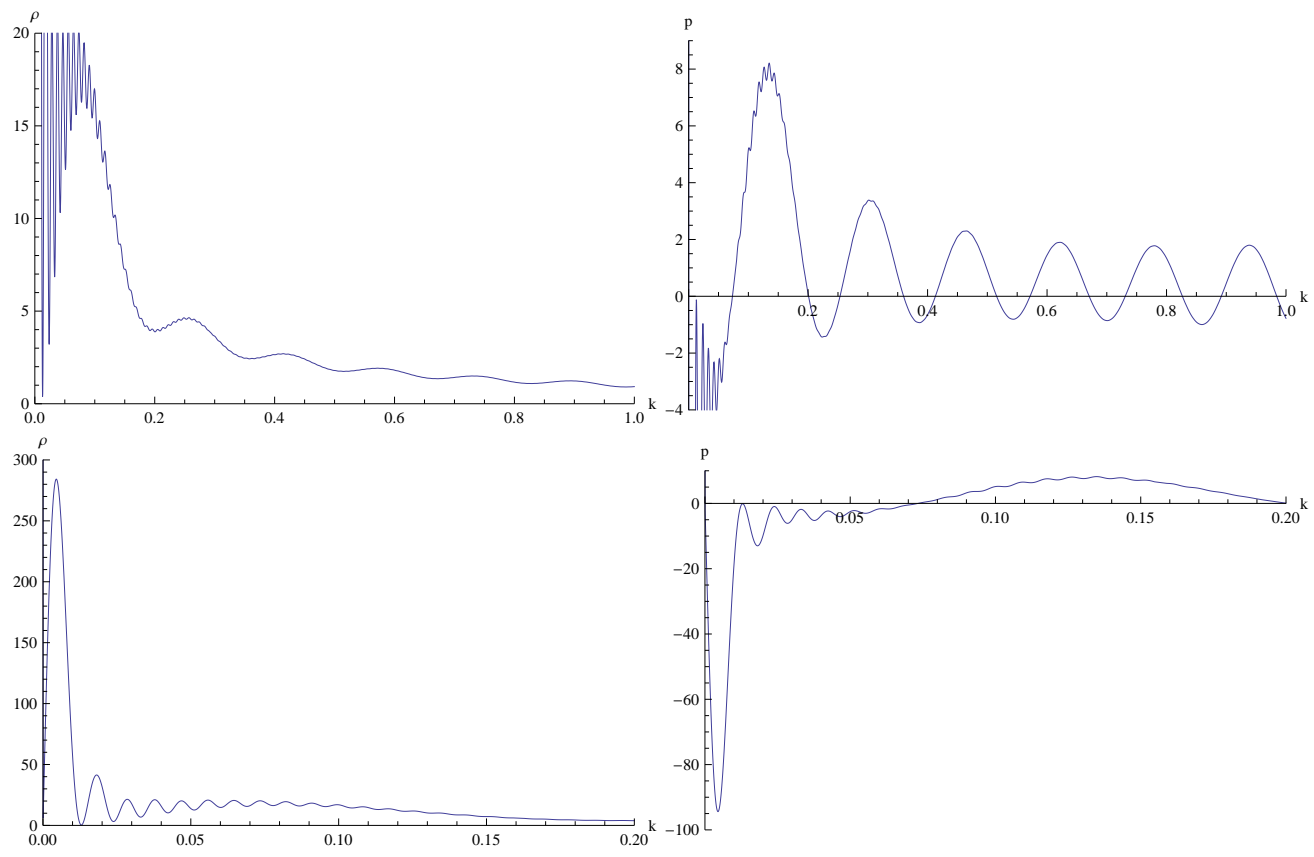

Figure 12: Integrands $\rho(k)$ and $p(k)$ in the RD period, caused by the double inflation model with the intermediate RD period. As in the third row of Figure 2, $\eta_{2}=-\eta_{1}=1$ and $\eta=20$ are taken. The other parameters are set to be $\eta_{2}^{\prime}=-\eta_{1}^{\prime}=40$ and $\eta_{3}^{\prime}=-\eta_{4}^{\prime}=160$. In the upper figures, the scales of the axes are taken to be same as those in the third row of Figure 2, while in the lower figures the IR region is magnified. 
We finally discuss natural values of the parameters in the model. If we set $\left|\eta_{4}^{\prime}\right| \sim \eta_{0}$, where $\eta_{0}$ is the present time, the large enhancement of (8.20) and (8.21) continues until $k \sim\left|\eta_{4}^{\prime}\right|^{-1} \sim \eta_{0}^{-1}$. Then the present energy density becomes the desired value of the order $\rho_{0} \sim\left(M_{P} H_{0}\right)^{2}$, if we set $H_{P} \sim M_{P}$. However, the modification of the wave function continues until $k \sim\left|\eta_{1}^{\prime}\right|^{-1}>$ $1.7 \times 10^{2}\left|\eta_{4}^{\prime}\right|^{-1} \sim 1.7 \times 10^{2} \eta_{0}^{-1}$. Since they are deep within the current horizon, they could be detected by observations. For instance, they may generate the CMB fluctuations, which contradict the observations.

If we set $\left|\eta_{1}^{\prime}\right|^{-1}<\eta_{0}^{-1}$ instead, the modes within the current horizon are not modified by the pre-inflation at all. However, the contribution of the large IR peak to the energy density terminates at a too small value of $k$, giving

$$
\rho_{0}^{\text {IRpeak }} \sim \frac{H_{P}^{2}}{8 \pi^{2} a_{0}^{2}} 2 \int_{0}^{\pi\left|4 \eta_{4}^{\prime}\right|^{-1}} d k k=\frac{H_{P}^{2}}{8 \pi^{2} a_{0}^{2}}\left(\frac{\pi}{4\left|\eta_{4}^{\prime}\right|}\right)^{2}<\frac{1}{512} H_{P} H_{I} H_{0}^{2},
$$

where we have approximated that the IR peak is located at $\pi\left|4 \eta_{4}^{\prime}\right|^{-1}$ and has form of an isosceles triangle with the slope given by (8.20). In the last inequality, $\left|\eta_{4}^{\prime}\right|^{-1}=\sqrt{\frac{H_{I}}{H_{P}}}\left|\eta_{1}^{\prime}\right|^{-1},\left|\eta_{1}^{\prime}\right|^{-1}<\eta_{0}^{-1}$, and $H_{0}=2\left(a_{0} \eta_{0}\right)^{-1}$ have been used. While it is enhanced from (7.22), it is still smaller than the desired value $\left(M_{P} H_{0}\right)^{2}$.

The above problem has been caused by the modes with $\left|\eta_{4}^{\prime}\right|^{-1}<k<\left|\eta_{1}^{\prime}\right|^{-1}$. The present model has the constraint $\left|\eta_{1}^{\prime}\right|^{-1} \gg\left|\eta_{4}^{\prime}\right|^{-1}$ given by (A.8). In other models with $\left|\eta_{1}^{\prime}\right|^{-1} \sim\left|\eta_{4}^{\prime}\right|^{-1}$, such as the one studied in Section 8.1, the problem is resolved.

\section{B IR behaviors of the wave functions}

In this appendix, we examine IR behaviors of the wave functions and confirm that the IR form (4.20) holds in all the periods in the cosmic history.

\section{B.1 Single inflation model}

We first study the case in the MD period in the single inflation model, studied in Section 4 Eq. (4.10) is rewritten as

$$
\left.\begin{array}{c}
\left(\begin{array}{l}
C \\
D
\end{array}\right)=\frac{-1}{2 k^{2} \eta_{4}^{2}}\left(\begin{array}{c}
{\left[1-e^{2 i k \eta_{4}} \sum_{n=3}^{\infty} \frac{1}{n !}\left(-2 i k \eta_{4}\right)^{n}\right] e^{-i k\left(\eta_{4}+\eta_{3}\right)}} \\
e^{-i k\left(\eta_{4}+\eta_{3}\right)}
\end{array}\right. \\
e^{i k\left(\eta_{4}+\eta_{3}\right)} \\
{\left[1-e^{-2 i k \eta_{4}} \sum_{n=3}^{\infty} \frac{1}{n !}\left(2 i k \eta_{4}\right)^{n}\right] e^{i k\left(\eta_{4}+\eta_{3}\right)}}
\end{array}\right)\left(\begin{array}{l}
A \\
B
\end{array}\right),
$$


where the terms with $k^{1}$ and $k^{2}$ cancel in the square bracket. Plugging in (4.21), one obtains

$$
\begin{aligned}
C= & \frac{1}{4 k^{4} \eta_{1}^{2} \eta_{4}^{2}}\left(\left[1-e^{-2 i k \eta_{1}} \sum_{n=3}^{\infty} \frac{1}{n !}\left(2 i k \eta_{1}\right)^{n}\right]\left[1-e^{2 i k \eta_{4}} \sum_{n=3}^{\infty} \frac{1}{n !}\left(-2 i k \eta_{4}\right)^{n}\right]\right. \\
\left.\times e^{i k\left(\eta_{1}+\eta_{2}-\eta_{3}-\eta_{4}\right)}-e^{-i k\left(\eta_{1}+\eta_{2}-\eta_{3}-\eta_{4}\right)}\right) & \\
D= & \frac{1}{4 k^{4} \eta_{1}^{2} \eta_{4}^{2}}\left(\left[1-e^{-2 i k \eta_{1}} \sum_{n=3}^{\infty} \frac{1}{n !}\left(2 i k \eta_{1}\right)^{n}\right] e^{i k\left(\eta_{1}+\eta_{2}-\eta_{3}-\eta_{4}\right)}\right. \\
& \left.-\left[1-e^{-2 i k \eta_{4}} \sum_{n=3}^{\infty} \frac{1}{n !}\left(2 i k \eta_{4}\right)^{n}\right] e^{-i k\left(\eta_{1}+\eta_{2}-\eta_{3}-\eta_{4}\right)}\right)
\end{aligned}
$$

Expanding in terms of $k$, one finds

$$
\begin{aligned}
C & =\frac{\eta_{1}+\eta_{2}-\eta_{3}-\eta_{4}}{2 \eta_{1}^{2} \eta_{4}^{2}} i k^{-3}+\mathcal{O}\left(k^{-1}\right), \\
D & =\frac{\eta_{1}+\eta_{2}-\eta_{3}-\eta_{4}}{2 \eta_{1}^{2} \eta_{4}^{2}} i k^{-3}+\mathcal{O}\left(k^{-1}\right)
\end{aligned}
$$

where the leading terms with $k^{-4}$ and also the terms with $k^{-2}$ cancel. The coefficients of $C$ and $D$ coincide at low powers of $k$, and begin to differ at the term with $k^{0}$ as

$$
C-D=\frac{\left(2 \eta_{1}+2 \eta_{2}-2 \eta_{3}+\eta_{4}\right) \eta_{4}}{3 \eta_{1}^{2}}+\mathcal{O}\left(k^{2}\right)
$$

where the terms with $k^{1}$ also cancel. By using the matching relations (3.6) and (3.9), the first term in (B.6) vanishes, and the second term gives

$$
C-D=\left(-\frac{4 \eta_{2} \eta_{4}}{9}+\frac{\eta_{4}^{4}}{180 \eta_{2}^{2}}\right) k^{2}+\mathcal{O}\left(k^{3}\right) \text {. }
$$

The wave function (4.6) is written as

$$
\chi_{\mathrm{MD}}=\frac{C+D}{2}\left(\chi_{\mathrm{BD}}+\chi_{\mathrm{BD}}^{*}\right)+\frac{C-D}{2}\left(\chi_{\mathrm{BD}}-\chi_{\mathrm{BD}}^{*}\right),
$$

where

$$
\begin{aligned}
\chi_{\mathrm{BD}}+\chi_{\mathrm{BD}}^{*} & =\frac{2}{\sqrt{2 k}}\left(\cos (k \eta)-\frac{\sin (k \eta)}{k \eta}\right) \\
& =\frac{2}{\sqrt{2 k}}\left(-\frac{1}{3}(k \eta)^{2}+\mathcal{O}\left((k \eta)^{4}\right)\right), \\
\chi_{\mathrm{BD}}-\chi_{\mathrm{BD}}^{*} & =\frac{-2 i}{\sqrt{2 k}}\left(\sin (k \eta)+\frac{\cos (k \eta)}{k \eta}\right) \\
& =\frac{-2 i}{\sqrt{2 k}}\left((k \eta)^{-1}+\mathcal{O}(k \eta)\right) .
\end{aligned}
$$


Then the first and second terms in (B.8) behave as $(C+D)\left(\chi_{\mathrm{BD}}+\chi_{\mathrm{BD}}^{*}\right) \sim$ $k^{-3} \cdot k^{3 / 2}=k^{-3 / 2}$ and $(C-D)\left(\chi_{\mathrm{BD}}-\chi_{\mathrm{BD}}^{*}\right) \sim k^{2} \cdot k^{-3 / 2}=k^{1 / 2}$ in the IR limit. From (B.4), (B.5), and (B.10), the next-to-leading-order term in $(C+D)\left(\chi_{\mathrm{BD}}+\right.$ $\chi_{\mathrm{BD}}^{*}$ ) has $k^{1 / 2}$. Hence, (B.8) behaves as

$$
\chi_{\mathrm{MD}}^{\mathrm{IR}}=\frac{\eta_{1}+\eta_{2}-\eta_{3}-\eta_{4}}{2 \eta_{1}^{2} \eta_{4}^{2}} i k^{-3} \cdot \frac{2}{\sqrt{2 k}}\left(-\frac{1}{3}\right)(k \eta)^{2}+\mathcal{O}\left(k^{1 / 2}\right)
$$

in the IR region. The leading term in (B.13) gives

$$
u_{\mathrm{MD}}^{\mathrm{IR}}=\frac{\eta_{1}+\eta_{2}-\eta_{3}-\eta_{4}}{2 \eta_{1}^{2} \eta_{4}^{2}} i k^{-3} \cdot \frac{2}{\sqrt{2 k}}\left(-\frac{1}{3}\right)(k \eta)^{2} \cdot \frac{2 \eta_{4} H_{I} \eta_{1}^{2}}{\eta^{2}}=\frac{i}{\sqrt{2}} H_{I} k^{-3 / 2}
$$

where (4.3) with (3.1), (3.5), and (3.8) has been used in the first equality. In the second equality, the matching relations (3.6) and (3.9) have been used. The same result with (4.20) is obtained again in the MD region. Note that the leading IR behavior $k^{-3 / 2}$ is much better than the naive estimation $k^{-11 / 2}$ mentioned above (4.21).

\section{B.2 Double inflation model with the intermediate CD stage}

We next study the case in the double inflation model with the intermediate CD stage, studied in Section 8.1.

In the pre-inflation period, the wave function (8.10) behaves as (8.19) in the IR region, as can be seen by the same argument in Section 4. In the CD period, by expanding (8.16) in terms of $k$, one obtains

$$
\begin{aligned}
\tilde{A} & =\left(\frac{i}{4} \frac{k}{\gamma}+\mathcal{O}\left(k^{2}\right)\right) e^{\gamma \tilde{\eta}_{2}} \\
\tilde{B} & =\left(i \frac{\gamma}{k}+\frac{i}{4}\left(1+2 \gamma \tilde{\eta}_{2}\right) \frac{k}{\gamma}+\mathcal{O}\left(k^{2}\right)\right) e^{-\gamma \tilde{\eta}_{2}} .
\end{aligned}
$$

Then the wave function (8.11) becomes

$$
\chi_{\mathrm{CD}}^{\mathrm{IR}}=i \frac{\gamma}{k} \frac{1}{\sqrt{2 k}} e^{\gamma\left(\eta-\tilde{\eta}_{2}\right)}+\mathcal{O}\left(k^{1 / 2}\right) .
$$

The leading term gives

$$
u_{\mathrm{CD}}^{\mathrm{IR}}=i \frac{\gamma}{k} \frac{1}{\sqrt{2 k}} e^{\gamma\left(\eta-\tilde{\eta}_{2}\right)} \frac{1}{e^{\gamma \eta}}=\frac{i}{\sqrt{2}} H_{P} k^{-3 / 2},
$$

where (4.3) with (8.1) has been used in the first equality. In the second equality, (8.2) and (8.6) have been used. Eq. (8.19) is obtained again.

In the subsequent inflation period, by plugging (8.16) into (8.17), and expanding in terms of $k$, one obtains

$$
\begin{aligned}
\tilde{C}+\tilde{D} & =\frac{i}{2} \frac{\gamma}{k}\left(e^{\gamma\left(\tilde{\eta}_{3}-\tilde{\eta}_{2}\right)}-e^{-\gamma\left(\tilde{\eta}_{3}-\tilde{\eta}_{2}\right)}\right)+\mathcal{O}\left(k^{0}\right), \\
\tilde{C}-\tilde{D} & =e^{\gamma\left(\tilde{\eta}_{3}-\tilde{\eta}_{2}\right)}+\mathcal{O}\left(k^{2}\right)
\end{aligned}
$$


Then, when the wave function (8.12) is rewritten as in (B.8), with $C$ and $D$ replaced by $\tilde{C}$ and $\tilde{D}$, the second term dominates over the first term, giving

$$
\chi_{\mathrm{Inf}}^{\mathrm{IR}}=\frac{1}{2} e^{\gamma\left(\tilde{\eta}_{3}-\tilde{\eta}_{2}\right)} \frac{-2 i}{\sqrt{2 k}}(k \eta)^{-1}+\mathcal{O}\left(k^{1 / 2}\right) .
$$

The leading term gives

$$
u_{\mathrm{Inf}}^{\mathrm{IR}}=\frac{1}{2} e^{\gamma\left(\tilde{\eta}_{3}-\tilde{\eta}_{2}\right)} \frac{-2 i}{\sqrt{2 k}}(k \eta)^{-1} \frac{1}{-1 /\left(H_{I} \eta\right)}=\frac{i}{\sqrt{2}} H_{P} k^{-3 / 2},
$$

where (4.3) with (8.1) has been used in the first equality, and (8.7) in the second equality. Then eq. 8.19) is obtained again.

In the subsequent RD period, by plugging (8.16) and (8.17) into (8.18), and expanding in terms of $k$, one obtains

$$
\begin{aligned}
A+B & =\frac{i}{6} \eta_{2} k\left[\left(4-3 \gamma \eta_{2}\right) e^{\gamma\left(\tilde{\eta}_{3}-\tilde{\eta}_{2}\right)}+3 \gamma \eta_{2} e^{-\gamma\left(\tilde{\eta}_{3}-\tilde{\eta}_{2}\right)}\right]+\mathcal{O}\left(k^{2}\right) \\
A-B & =-\left(\eta_{2} k\right)^{-2} e^{\gamma\left(\tilde{\eta}_{3}-\tilde{\eta}_{2}\right)}+\mathcal{O}\left(k^{0}\right)
\end{aligned}
$$

Then, in the wave function (4.5), rewritten as

$$
\begin{aligned}
\chi_{\mathrm{RD}} & =\frac{A+B}{2}\left(\chi_{\mathrm{PW}}+\chi_{\mathrm{PW}}^{*}\right)+\frac{A-B}{2}\left(\chi_{\mathrm{PW}}-\chi_{\mathrm{PW}}^{*}\right) \\
& =\frac{A+B}{2} \frac{2}{\sqrt{2 k}} \cos (k \eta)+\frac{A-B}{2} \frac{-2 i}{\sqrt{2 k}} \sin (k \eta),
\end{aligned}
$$

the second term dominates over the first term, giving

$$
\chi_{\mathrm{RD}}^{\mathrm{IR}}=-\frac{1}{2}\left(\eta_{2} k\right)^{-2} e^{\gamma\left(\tilde{\eta}_{3}-\tilde{\eta}_{2}\right)} \frac{-2 i}{\sqrt{2 k}} k \eta+\mathcal{O}\left(k^{1 / 2}\right) .
$$

The leading term gives

$$
u_{\mathrm{RD}}^{\mathrm{IR}}=-\frac{1}{2}\left(\eta_{2} k\right)^{-2} e^{\gamma\left(\tilde{\eta}_{3}-\tilde{\eta}_{2}\right)} \frac{-2 i}{\sqrt{2 k}} k \eta \frac{1}{\alpha \eta}=\frac{i}{\sqrt{2}} H_{P} k^{-3 / 2},
$$

where (4.3) with (3.1) has been used in the first equality. In the second equality, (3.5), (3.6), and (8.7) have been used. Eq. (8.19) is obtained again.

In the subsequent MD period, by plugging (8.16), (8.17), and (8.18) into (4.10), and expanding in terms of $k$, one obtains

$$
\begin{aligned}
C+D= & -\frac{3 i}{2 \eta_{2}^{2} \eta_{4}} e^{\gamma\left(\tilde{\eta}_{3}-\tilde{\eta}_{2}\right)} k^{-3}+\mathcal{O}\left(k^{-1}\right) \\
C-D= & \frac{\eta_{4}}{180 \eta_{2}^{2} \gamma^{3}}\left[\left(60\left(\gamma \eta_{2}\right)^{4}-80\left(\gamma \eta_{2}\right)^{3}+\left(\gamma \eta_{4}\right)^{3}\right) e^{\gamma\left(\tilde{\eta}_{3}-\tilde{\eta}_{2}\right)}\right. \\
& \left.-60\left(\gamma \eta_{2}\right)^{4} e^{-\gamma\left(\tilde{\eta}_{3}-\tilde{\eta}_{2}\right)}\right] k^{2}+\mathcal{O}\left(k^{3}\right)
\end{aligned}
$$

Then, when the wave function (4.6) is written as (B.8), the first term dominates over the second term, giving

$$
\chi_{\mathrm{MD}}^{\mathrm{IR}}=\frac{1}{2} \frac{-3 i}{2 \eta_{2}^{2} \eta_{4}} e^{\gamma\left(\tilde{\eta}_{3}-\tilde{\eta}_{2}\right)} k^{-3} \frac{2}{\sqrt{2 k}}\left(-\frac{1}{3}\right)(k \eta)^{2}+\mathcal{O}\left(k^{1 / 2}\right) .
$$


The leading term gives

$$
u_{\mathrm{MD}}^{\mathrm{IR}}=\frac{1}{2} \frac{-3 i}{2 \eta_{2}^{2} \eta_{4}} e^{\gamma\left(\tilde{\eta}_{3}-\tilde{\eta}_{2}\right)} k^{-3} \frac{2}{\sqrt{2 k}}\left(-\frac{1}{3}\right)(k \eta)^{2} \frac{1}{\beta \eta^{2}}=\frac{i}{\sqrt{2}} H_{P} k^{-3 / 2},
$$

where (4.3) with (3.1) has been used in the first equality. In the second equality, we have used (8.7) and $2 \beta \eta_{2}^{2} \eta_{4}=H_{I}^{-1}$, which is obtained by (3.5), (3.8), and (3.6). Then eq. (8.19) is obtained again.

\section{B.3 Double inflation model with the intermediate RD stage}

We finally study the case in the double inflation model with the intermediate RD stage, studied in Appendix A.

In the pre-inflation and pre-RD periods, the wave functions (8.10) and (A.10) behave as (8.19) in the IR region, as can be seen by the same calculations in Section 4 ,

In the inflation period, since (A.12) has the same form as (4.10) with primes added, the coefficients $C^{\prime}$ and $D^{\prime}$ behave again as (B.4), (B.5), and (B.6) in the IR regions. However, because of the matching relations (A.6) and (A.7), the leading term in (B.4) and (B.5) vanishes, but that in (B.6) does not vanish in this case. Then the second term dominates over the first term in (B.8). The leading term gives

$$
u_{\mathrm{Inf}}^{\mathrm{IR}}=\frac{1}{2} \frac{\left(2 \eta_{1}+2 \eta_{2}-2 \eta_{3}+\eta_{4}\right) \eta_{4}}{3 \eta_{1}^{2}} \frac{-2 i}{\sqrt{2 k}}(k \eta)^{-1}\left(-H_{I} \eta\right)=\frac{i}{\sqrt{2}} H_{P} k^{-3 / 2},
$$

where (B.6), (B.12), and (4.3) with (A.1) have been used in the first equality. In the second equality, the matching relations (A.6), (A.7), and (A.8) have been used. Eq. (8.19) is obtained again.

In the subsequent RD period, by plugging (A.11) and (A.12) into (8.18), and expanding in terms of $k$, one obtains

$$
\begin{aligned}
& A-B=\frac{2 \eta_{1}^{3}\left(\eta_{1}^{\prime}+\eta_{2}^{\prime}-\eta_{3}^{\prime}-\eta_{4}^{\prime}\right)-\eta_{4}^{\prime 3}\left(2 \eta_{1}^{\prime}+2 \eta_{2}^{\prime}-2 \eta_{3}^{\prime}+\eta_{4}^{\prime}\right)}{3 \eta_{1}^{2} \eta_{1}^{\prime 2} \eta_{4}^{\prime 2}} k^{-2}+\mathcal{O}\left(k^{0}\right) \\
& \begin{aligned}
A+B= & \frac{-1}{3 \eta_{1}^{2} \eta_{1}^{\prime 2} \eta_{4}^{\prime 2}}\left[\eta_{1}^{3}\left(\eta_{1}-2 \eta_{2}\right)\left(\eta_{1}^{\prime}+\eta_{2}^{\prime}-\eta_{3}^{\prime}-\eta_{4}^{\prime}\right)\right. \\
& \left.+\left(\eta_{1}+\eta_{2}\right)\left(2 \eta_{1}^{\prime}+2 \eta_{2}^{\prime}-2 \eta_{3}^{\prime}+\eta_{4}^{\prime}\right) \eta_{4}^{\prime 3}\right] i k^{-1}+\mathcal{O}\left(k^{1}\right)
\end{aligned}
\end{aligned}
$$

The leading terms with $k^{-6}$ to $k^{-3}$ have canceled, which can be checked, for instance, by rewriting (A.11), (A.12), and (8.18) as in (4.21) and (B.1). Substituting the matching relations (3.6), (A.6), and (A.7), Eqs. (B.34) and (B.35) 
become

$$
\begin{aligned}
& A-B=-\frac{\eta_{4}^{\prime 2}}{\eta_{1}^{2} \eta_{1}^{\prime 2}} k^{-2}+\mathcal{O}\left(k^{0}\right), \\
& A+B=\mathcal{O}\left(k^{1}\right) .
\end{aligned}
$$

Then, when the wave function (4.5) is rewritten as (B.26), the second term dominates over the first term and gives

$$
\chi_{\mathrm{RD}}^{\mathrm{IR}}=\frac{1}{2} \frac{-\eta_{4}^{\prime 2}}{\eta_{1}^{2} \eta_{1}^{\prime 2}} k^{-2} \frac{-2 i}{\sqrt{2 k}} k \eta+\mathcal{O}\left(k^{1 / 2}\right) .
$$

The leading term gives

$$
u_{\mathrm{RD}}^{\mathrm{IR}}=\frac{1}{2} \frac{-\eta_{4}^{\prime 2}}{\eta_{1}^{2} \eta_{1}^{\prime 2}} k^{-2} \frac{-2 i}{\sqrt{2 k}} k \eta \frac{1}{\alpha \eta}=\frac{i}{\sqrt{2}} H_{P} k^{-3 / 2},
$$

where (4.3) with (3.1) has been used in the first equality. In the second equality, (3.5) and (A.8) have been used. Eq. (8.19) is obtained again.

In the subsequent MD period, by plugging (A.11), (A.12), and (8.18) into (4.10), and expanding in terms of $k$, one obtains

$$
\begin{aligned}
C+D= & \frac{1}{3 \eta_{1}^{2} \eta_{4}^{2} \eta_{1}^{\prime 2} \eta_{4}^{\prime 2}}\left[\eta_{1}^{3}\left(\eta_{1}-2 \eta_{2}+2 \eta_{3}+2 \eta_{4}\right)\left(\eta_{1}^{\prime}+\eta_{2}^{\prime}-\eta_{3}^{\prime}-\eta_{4}^{\prime}\right)\right. \\
& \left.+\left(\eta_{1}+\eta_{2}-\eta_{3}-\eta_{4}\right)\left(2 \eta_{1}^{\prime}+2 \eta_{2}^{\prime}-2 \eta_{3}^{\prime}+\eta_{4}^{\prime}\right) \eta_{4}^{\prime 3}\right] i k^{-3} \\
& +\mathcal{O}\left(k^{-1}\right), \\
C-D= & \frac{\eta_{4}}{9 \eta_{1}^{2} \eta_{1}^{\prime 2} \eta_{4}^{\prime 2}}\left[2 \eta_{1}^{3}\left(\eta_{1}-2 \eta_{2}+2 \eta_{3}-\eta_{4}\right)\left(\eta_{1}^{\prime}+\eta_{2}^{\prime}-\eta_{3}^{\prime}-\eta_{4}^{\prime}\right)\right. \\
& \left.+\left(2 \eta_{1}+2 \eta_{2}-2 \eta_{3}+\eta_{4}\right)\left(2 \eta_{1}^{\prime}+2 \eta_{2}^{\prime}-2 \eta_{3}^{\prime}+\eta_{4}^{\prime}\right) \eta_{4}^{\prime 3}\right] k^{0} \\
& +\mathcal{O}\left(k^{2}\right) .
\end{aligned}
$$

Substituting the matching relations (3.6), (3.9), (A.6), and (A.7), Eqs. (B.40) and (B.41) become

$$
\begin{aligned}
C+D & =-\frac{3 \eta_{4}^{\prime 2}}{2 \eta_{1}^{2} \eta_{4} \eta_{1}^{\prime 2}} i k^{-3}+\mathcal{O}\left(k^{-1}\right) \\
C-D & =\mathcal{O}\left(k^{2}\right)
\end{aligned}
$$

Then in the wave function (B.8), the first term dominates over the second term, giving

$$
\chi_{\mathrm{MD}}^{\mathrm{IR}}=\frac{1}{2} \frac{-3 \eta_{4}^{\prime 2}}{2 \eta_{1}^{2} \eta_{4} \eta_{1}^{\prime 2}} i k^{-3} \frac{2}{\sqrt{2 k}}\left(-\frac{1}{3}\right)(k \eta)^{2}+\mathcal{O}\left(k^{1 / 2}\right)
$$


The leading term gives

$$
u_{\mathrm{MD}}^{\mathrm{IR}}=\frac{1}{2} \frac{-3 \eta_{4}^{\prime 2}}{2 \eta_{1}^{2} \eta_{4} \eta_{1}^{\prime 2}} i k^{-3} \frac{2}{\sqrt{2 k}}\left(-\frac{1}{3}\right)(k \eta)^{2} \frac{1}{\beta \eta^{2}}=\frac{i}{\sqrt{2}} H_{P} k^{-3 / 2},
$$

where (4.3) with (3.1) has been used in the first equality. In the second equality, we have used (A.8) and $2 \beta \eta_{1}^{2} \eta_{4}=H_{I}^{-1}$, which is obtained by (3.5) and (3.8) . Then eq. (8.19) is obtained again.

\section{References}

[1] P. A. R. Ade et al. [Planck Collaboration], arXiv:1303.5076 [astro-ph.CO].

[2] S. Weinberg, Rev. Mod. Phys. 61, 1 (1989).

[3] S. Weinberg, Phys. Rev. Lett. 59, 2607 (1987).

[4] N. D. Birrell and P. C. W. Davies, "Quantum Fields in Curved Space," Cambridge University Press, 1984.

[5] T. S. Bunch and P. C. W. Davies, Proc. Roy. Soc. Lond. A 360, 117 (1978).

[6] J. S. Dowker and R. Critchley, Phys. Rev. D 13, 3224 (1976).

[7] P. A. R. Ade et al. [Planck Collaboration], arXiv:1303.5082 [astro-ph.CO].

[8] P. R. Anderson, W. Eaker, S. Habib, C. Molina-Paris and E. Mottola, Phys. Rev. D 62, 124019 (2000) gr-qc/0005102.

[9] V. Mukhanov, "Physical foundations of cosmology," Cambridge, UK: Univ. Pr. (2005) $421 \mathrm{p}$

[10] V. F. Mukhanov, L. R. W. Abramo and R. H. Brandenberger, Phys. Rev. Lett. 78, 1624 (1997) gr-qc/9609026.

[11] L. R. W. Abramo, R. H. Brandenberger and V. F. Mukhanov, Phys. Rev. D 56, 3248 (1997) gr-qc/9704037.

[12] L. R. W. Abramo, Phys. Rev. D 60, 064004 (1999) astro-ph/9903270.

[13] I. A. Brown, L. Schrempp and K. Ananda, JCAP 1002, 011 (2010) arXiv:0909.1922 [gr-qc]].

[14] D. Glavan, T. Prokopec and V. Prymidis, Phys. Rev. D 89, 024024 (2014) arXiv:1308.5954 [gr-qc]]. 
[15] E. W. Kolb, S. Matarrese, A. Notari and A. Riotto, Phys. Rev. D 71, 023524 (2005) hep-ph/0409038].

[16] E. Barausse, S. Matarrese and A. Riotto, Phys. Rev. D 71, 063537 (2005) astro-ph/0501152.

[17] S. R. Coleman and F. De Luccia, Phys. Rev. D 21, 3305 (1980).

[18] S. W. Hawking and I. G. Moss, Phys. Lett. B 110, 35 (1982).

[19] A. D. Linde, Phys. Lett. B 175, 395 (1986).

[20] A. A. Starobinsky, Phys. Lett. B 91, 99 (1980).

[21] S. Singh, S. K. Modak and T. Padmanabhan, Phys. Rev. D 88, 125020 (2013) arXiv:1308.4976 [gr-qc]].

[22] A. D. Linde, Phys. Lett. B 116, 335 (1982).

[23] A. A. Starobinsky, Phys. Lett. B 117, 175 (1982).

[24] A. Vilenkin and L. H. Ford, Phys. Rev. D 26, 1231 (1982).

[25] A. D. Linde, "Particle physics and inflationary cosmology" Harwood, Chur, Switzerland, 1990, hep-th/0503203.

[26] F. Finelli, G. Marozzi, G. P. Vacca and G. Venturi, Phys. Rev. D 65, 103521 (2002) gr-qc/0111035.

[27] F. Finelli, G. Marozzi, A. A. Starobinsky, G. P. Vacca and G. Venturi, Phys. Rev. D 79, 044007 (2009) arXiv:0808.1786 [hep-th]].

[28] F. Finelli, G. Marozzi, A. A. Starobinsky, G. P. Vacca and G. Venturi, Phys. Rev. D 82, 064020 (2010) arXiv:1003.1327 [hep-th]].

[29] G. Marozzi, M. Rinaldi and R. Durrer, Phys. Rev. D 83, 105017 (2011) arXiv:1102.2206 [astro-ph.CO]].

[30] B. S. DeWitt, Phys. Rept. 19, 295 (1975).

[31] L. Parker and S. A. Fulling, Phys. Rev. D 9, 341 (1974).

[32] T. S. Bunch, J. Phys. A 13, 1297 (1980).

[33] P. R. Anderson and L. Parker, Phys. Rev. D 36, 2963 (1987).

[34] H. Kitamoto and Y. Kitazawa, Phys. Rev. D 85, 044062 (2012) arXiv:1109.4892 [hep-th]]. 
[35] H. Kitamoto and Y. Kitazawa, arXiv:1402.2443 [hep-th].

[36] H. Aoki and S. Iso, Phys. Rev. D 86, 013001 (2012) arXiv:1201.0857 [hep$\mathrm{ph}]$.

[37] L. D. Landau and E. M. Lifschits, "Quantum Mechanics (Non-Relativistic Theory): Course of Theoretical Physics, Volume 3,"

[38] R. K. Jain, P. Chingangbam, J. -O. Gong, L. Sriramkumar and T. Souradeep, JCAP 0901, 009 (2009) [arXiv:0809.3915 [astro-ph]].

[39] R. K. Jain, P. Chingangbam, L. Sriramkumar and T. Souradeep, Phys. Rev. D 82, 023509 (2010) [arXiv:0904.2518 [astro-ph.CO]].

[40] B. Freivogel, Y. Sekino, L. Susskind and C. -P. Yeh, Phys. Rev. D 74, 086003 (2006) hep-th/0606204.

[41] Y. Habara, H. Kawai, M. Ninomiya and Y. Sekino, Phys. Lett. B 707, 198 (2012) arXiv:1103.0299 [hep-th]].

[42] Y. Habara, H. Kawai, M. Ninomiya and Y. Sekino, Phys. Rev. D 85, 104027 (2012) arXiv:1110.5392 [hep-th]].

[43] T. Tanaka and Y. Urakawa, Class. Quant. Grav. 30, 233001 (2013) arXiv:1306.4461 [hep-th]].

[44] D. Marolf and I. A. Morrison, Gen. Rel. Grav. 43, 3497 (2011) arXiv:1104.4343 [gr-qc]]. 